\title{
Markov State and Diffusive Stochastic Models in Electron Spin Resonance
}

\author{
Deniz Sezer and Benoît Roux
}

\subsection{Introduction}

Continuous-wave electron spin resonance (cwESR) experiments combined with site-directed spin labeling (SDSL) techniques are a rich source of information about the local structure and dynamics of biomolecules in native-like environments $[6,10,16]$. Partly owing to its high sensitivity and the relative ease with which biomolecules can be systematically labeled at almost any desired location (e.g., by introducing cysteine mutations in proteins), ESR plays an increasingly important role in studies of proteins [26], nucleic acids [27], and membrane systems [37]. An exciting aspect of cw-ESR is the extreme sensitivity of the technique to the details of the dynamical processes occurring at the molecular level. Cw-ESR spectra are sensitive to dynamics over a broad range of time scales, from hundreds of picoseconds to tens of nanoseconds, depending on the strength of the constant magnetic field or the corresponding microwave frequency. While the slower end of this temporal range is routinely covered by lower fields/frequencies, like the most commonly used X-band, the faster end has recently become accessible as a result of vigorous

\section{Sezer $(\varangle)$}

Faculty of Engineering and Natural Sciences, Sabancı University, Istanbul, Turkey

e-mail: dsezer@sabanciuniv.edu

\section{B. Roux}

Department of Biochemistry and Molecular Biology,

The University of Chicago, Chicago, USA

e-mail:roux@uchicago.edu developments in high-field/high-frequency ESR $[5,13,20]$. However, despite the great progress with the experimental cw-ESR and SDSL techniques, the development of a comprehensive set of theoretical methods able to account quantitatively for all the spectral features in terms of atomic models remains challenging.

A qualitative interpretation of cw-ESR spectra is relatively straightforward when the overall purpose of an experimental investigation is to delineate broad structural features of a protein. For instance, secondary structural elements can be mapped out by systematically scanning the protein by SDSL, and comparing the resulting spectra. Information about the overall position of the subunits of a membrane protein relative to the lipid bilayer can also be obtained with the aid of additional water- or membrane-soluble paramagnetic agents. The situation is very different when detailed structural and dynamic information about a system is sought, and the specific features of the spectral line shapes need to be properly interpreted. In this case, understanding the link between the conformational dynamics of the spin label and the observed spectra becomes of paramount importance. Unambiguously inferring the underlying molecular processes from spectra is difficult, however, even when using high-field/high-frequency ESR. The task becomes particularly challenging for spinlabeled biomolecules because they undergo complex motions occurring over a multitude of overlapping time scales. The internal dynamics of the spectroscopic reporter, i.e., the spin label, adds 
another layer of complexity that must also be accounted for.

Currently, the most established theoretical/ computational method for quantifying cw-ESR spectra is based on the stochastic Liouville equation (SLE). Spectral line shapes are simulated by generating a matrix representation for the Liouville operator corresponding to the relevant spin dynamics coupled to the hypothesized stochastic processes [19, 46]. Among the various stochastic models, most sophisticated are the MOMD (microscopic order macroscopic disorder) [38] and SRLS (slowly relaxing local structure) [40, 41] models, in which the dynamics of the spin label relative to the magnetic fields applied in the laboratory is described as a collection of nested rotational transformations evolving in a diffusive manner (see Sect. 10.2.2). The necessary matrix diagonalization and simulation of cw-ESR spectra is done in a very efficient way by the SLE numerical solver-a suite of programs for simulating and fitting slow-motional ESR spectradeveloped in the laboratory of Jack Freed [7]. The outcome of an SLE analysis is typically a small set of phenomenological parameters associated with the rate and the range of motion of the spin label in some local mean-field potential. In practice it is necessary to keep the internal structure of the phenomenological stochastic model relatively simple to maintain the size of the problems within approachable numerical limits for the SLE solver. To avoid this issue, an alternative route is to bypass the construction of SLE altogether and simulate $\mathrm{cw}$-ESR spectra directly from stochastic trajectories [14, 15, 17, 44, 52, 55]. The most important limitation of such phenomenological approaches, which thwarts developing insight about the molecular factors reported by the details of the spectra, remains the difficulty to understand the correspondence between the fitted mean-field parameters of the stochastic model and the underlying atomistic motions.

In this regard, all-atom molecular dynamics (MD) simulations with explicit solvent offer, perhaps, one of the most promising approaches for calculating cw-ESR spectra directly without extra assumptions about phenomenological models [4, 12, 30, 53]. In principle, MD simulations provide a "virtual route" to unambiguously link the atomistic dynamics to the experimentally observed cw-ESR spectra. Such an approach is, after all, routinely used to analyze and interpret results from nuclear magnetic resonance (NMR) [34]. However, a straightforward all-atom MD strategy for calculating ESR spectra remains challenging, even with current computational resources [47, 48]. The reason for this is both simple and complex. In NMR, the spin of the nuclei are only weakly coupled to their surrounding, therefore most magnetic relaxation coefficients can be calculated accurately from nanosecond trajectories using Redfield theory [43], which follows from time-dependent perturbation theory in quantum mechanics carried to second order. In contrast, the coupling of an electron spin to its environment is almost three orders of magnitude stronger than the coupling of nuclear spins. As a result, in most ESR experiments with spinlabeled macromolecules a perturbative treatment is not applicable. For this reason, the quantal degrees of freedom must be propagated for hundreds of nanoseconds to calculate spectra with a reasonable resolution of detail. Paradoxically, all issues of statistical convergence are not immediately resolved even when one trajectory is sufficiently long to allow the spin label to explore all accessible configurations and lose its correlation. The problem is that a large number of independent "samples" are necessary for a reliable estimate of the ESR spectrum. To clarify this point, it is useful to consider that the effective error of an ensemble average normally goes as $\sigma^{2} / \sqrt{N}$, where $N$ is the number of independent samples and $\sigma^{2}$ is the intrinsic variance of the signal. When the averaging process is carried out from a trajectory of total length $\mathscr{T}$ the number of independent samples is typically understood as $\mathscr{T} / \tau_{c}$, where $\tau_{c}$ is the correlation time. In the case of biomolecular ESR, $\tau_{c}$ can be on the order of tens or hundreds of nanoseconds, which does not correspond to exceedingly long trajectories with current standards. However, due to the strong coupling of the electron spin to the orientation of the nitroxide label, the effective $\sigma^{2}$ of cw-ESR spectra in the presence of such slow motions is very large. As a consequence, small changes in one classical trajectory of length $T$ can lead to 
considerable variations in the resulting spectrum. For this reason, one needs a very large $N$ to get a reliable spectrum. If the averaging process is carried out from an ensemble, then a large number of sample trajectories is required. Alternatively, if the averaging process is carried out from a single trajectory then the latter has to be much longer than a single correlation time.

With the aim of establishing a flexible computational formalism for simulating cw-ESR spectra, we have developed a framework that circumvents these difficulties. Relevant information about the spin-label dynamics is first extracted from (relatively short) MD trajectories and mapped onto a Markov state model (MSM). Extremely long and computationally inexpensive stochastic state-hoping trajectories are then generated, while global tumbling of the macromolecule can be incorporated via a rotational diffusion model. Finally, the quantal degrees of freedom can be propagated along these trajectories to calculate cw-ESR spectra accurately [47-49]. The feasibility of this approach was demonstrated in Ref. [50], where it was successfully applied to the simulation of multifrequency spectra of spinlabeled T4 Lysozyme [56]. In this chapter, we review the main theoretical and practical elements of the method.

\subsection{General Overview}

Before discussing the details of the approach, we start by giving a quick overview of the problem. To this end, we first introduce the quantum mechanical aspect of the problem (Sect. 10.2.1), then look at various models of the classical molecular motion (Sect. 10.2.2), and finally combine the two by illustrating the effect of molecular tumbling on cw-ESR spectra (Sect. 10.2.3). This structure reflects the overall organization of the chapter: Sect. 10.3 is concerned with the MSM modeling of the classical dynamics of a proteinattached spin label, Sect. 10.4 presents the details of the quantal spin dynamics, and Sect. 10.5 discusses the combination of the two aspects. Simulations of multifrequency cw-ESR spectra of spin-labeled T4 Lysozyme (Sect. 10.5.3) demonstrate the power of this novel methodology in practical applications.

\subsubsection{The Nitroxide Spin Hamiltonian}

Cw-ESR spectroscopy consists in measuring the transverse magnetization from a bulk system in which nitroxide spin labels have been introduced. A nitroxide has an unpaired electron of spin $S=$ $1 / 2$ and a nitrogen nucleus with spin $I=1$ (for $\left.{ }^{14} \mathrm{~N}\right)$ or $I=1 / 2\left(\right.$ for $\left.{ }^{15} \mathrm{~N}\right)$. The spin Hamiltonian of the nitroxide spin label, accounting for the interactions of the electron and nuclear spins, is

$$
\hat{H}(t)=\left|\gamma_{\mathrm{e}}\right|[\mathbf{B} \cdot \mathbf{G}(t) \cdot \hat{\mathbf{S}}+\hat{\mathbf{I}} \cdot \mathbf{A}(t) \cdot \hat{\mathbf{S}}]
$$

in units of angular frequency. (Bold letters are used to denote vectors and matrices in physical space, Hilbert space operators are indicated with a caret.) Here, $\gamma_{\mathrm{e}}=-1.76086 \times$ $10^{-2} \mathrm{rad} \mathrm{ns}^{-1} \mathrm{G}^{-1}$ is the electron gyromagnetic ratio, $\hat{\mathbf{S}}$ and $\hat{\mathbf{I}}$ are the electron and nuclear spin operators, $\mathbf{A}$ is the hyperfine tensor (expressed in units of magnetic field) and

$$
\mathbf{G}(t) \equiv \mathbf{g}(t) / g_{\mathrm{e}}
$$

is the electronic $g$ tensor, $\mathbf{g}$, divided by the free electron $g$-factor, $g_{\mathrm{e}}=2.0023193$. The electron Zeeman interaction and the electron-nucleus hyperfine interaction are explicitly accounted for in the Hamiltonian (10.1). In contrast, the nuclear Zeeman and quadrupolar (in the case of $I=1$ ) interactions have been neglected.

To a very good approximation, the coupling tensors $\mathbf{G}$ and $\mathbf{A}$ are diagonal in the same nitroxide-fixed coordinate frame $\mathrm{N}$. The standard choice of axes in $\mathrm{N}$ with respect to the nitroxide structure is shown in Fig. 10.1. Typical magnetic tensor values for nitroxide spin labels on biomolecules are

$$
\begin{aligned}
\mathbf{g}^{\mathrm{N}} & =\operatorname{diag}(2.008,2.006,2.0022), \\
\mathbf{A}^{\mathrm{N}} & =\operatorname{diag}(5.0,5.0,37.0) \text { Gauss. }
\end{aligned}
$$

In this picture, the explicit time dependence of the magnetic tensors in (10.1) is due to the classical rotational dynamics of the coordinate system $\mathrm{N}$ with respect to the stationary laboratory 


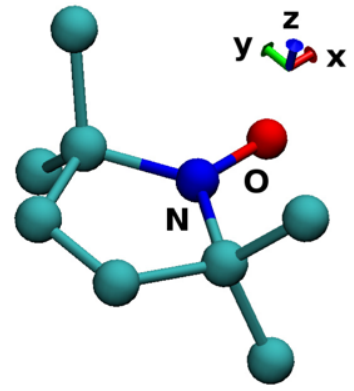

Fig. 10.1 The direction of the principle axes in the coordinate system $\mathrm{N}$ attached to the nitroxide ring. The $x$ direction is along the $\mathrm{N}-\mathrm{O}$ bond, the $z$ direction is perpendicular to the ring, and the $y$ direction is such that a right-handed system of axes is formed

frame $\mathrm{L}$, in which the constant magnetic field $\mathbf{B}=$ $(0,0, B)$ is applied. ${ }^{1}$ Since the spins are quantized along $\mathbf{B}$ all the vector and tensor components in the Hamiltonian are with respect to $L$.

The dynamics that cw-ESR experiments aim to probe leaves its mark on the spin dynamics described above by modulating the components $G_{i j}^{\mathrm{L}}$ and $A_{i j}^{\mathrm{L}}(i, j=x, y, z)$ of the Zeeman and hyperfine magnetic tensors in the laboratory frame. Since these tensors remain unchanged in a coordinate system fixed on the nitroxide (cf. Fig. 10.1), the molecular motion is encoded in the form of rotation matrices $R^{\mathrm{LN}}(t)$ that transform the tensor components from the $\mathrm{N}$ to the $\mathrm{L}$ system of axes according to

$$
\begin{aligned}
G_{i j}^{\mathrm{L}}(t) & =\sum_{k=x, y, z} R_{i k}^{\mathrm{LN}}(t) G_{k k}^{\mathrm{N}} R_{j k}^{\mathrm{LN}}(t), \\
A_{i j}^{\mathrm{L}}(t) & =\sum_{k} R_{i k}^{\mathrm{LN}}(t) A_{k k}^{\mathrm{N}} R_{j k}^{\mathrm{LN}}(t) .
\end{aligned}
$$

The time dependence of the transformation matrices is due to the dynamics of the nitroxide, as well as the global and internal dynamics of

\footnotetext{
${ }^{1}$ Although not rigorously correct, the assumption that the classical dynamics is completely uninfluenced by the states of the quantum system is typically an excellent approximation for room-temperature magnetic resonance. One minor inconvenience is that the equilibrium population of the states of the spin system corresponds to an infinite temperature. This, however, affects only the longitudinal magnetization but not the transverse magnetization whose evolution is calculated.
}

the molecule to which the spin label is covalently attached. Different motional models assumed for these dynamical modes result in different sequences $R^{\mathrm{LN}}(t)$, as discussed next.

\subsubsection{Stochastic Models of Molecular Motion}

The transformation from the nitroxide-fixed to the laboratory system of coordinate axes can be achieved as a sequence of nested rotational transformations. For example, the MOMD model mentioned in Sect. 10.1 can be illustrated schematically as

$$
\mathrm{L} \stackrel{\text { powder }}{\longrightarrow} \mathrm{M} \stackrel{\begin{array}{c}
\text { restricted } \\
\text { diffusion }
\end{array}}{\longrightarrow} \mathrm{S} \stackrel{\text { fixed }}{\longrightarrow} \mathrm{N} \text {. }
$$

Here $S$ refers to the system of coordinate axes fixed on the spin label and $M$ to the coordinate system attached to the macromolecule (e.g., protein) to which the spin label is covalently bonded. The model (10.5) represents the dynamics of the spin label with respect to the protein as restricted rotational diffusion. The coordinate system $S$ attached to the spin label is defined by the principle axes of its diffusion tensor. In general, these axes do not need to overlap with the principle axes of the magnetic tensors, defining the coordinate frame $\mathrm{N}$. The model (10.5) can therefore account for the possibility that the nitroxide frame $\mathrm{N}$ has a fixed, time-independent orientation with respect to $S$. In addition to the spin-label dynamics relative to the protein, in (10.5) the protein is allowed to be randomly orientated with respect to the laboratory frame. This would be the case for a large, relatively immobilized macromolecule in solution or a frozen (powder) sample. ${ }^{2}$

Clearly, more complex motional models can be constructed by combining independent or coupled nested rotations. Very attractive, however, is

\footnotetext{
${ }^{2} \mathrm{~A}$ time-dependent or constant rotation matrix is associated with each successive transformation in a motional model like (10.5). The matrix for the net transformation from $\mathrm{L}$ to $\mathrm{N}$, to be employed in (10.4), is obtained as the product of the successive rotation matrices: $R^{\mathrm{LN}}(t)=$ $R^{\mathrm{LM}} R^{\mathrm{MS}}(t) R^{\mathrm{SN}}$.
} 
the alternative to forgo completely any stochastic model, and use the time-dependent dynamics of $\mathrm{N}$ relative to $\mathrm{L}$ extracted directly from atomistic MD simulations. This approach has been pursued by many [4, 8, 12, 22, 30, 53], following the pioneering work of Steinhoff and Hubbell from more than a decade and a half ago [52]. In this approach, the time-dependent rotation matrices $R^{\mathrm{LN}}(t)$ can be obtained directly from the snapshots of the classical MD simulations, which can be represented as

$$
\mathrm{L} \stackrel{\text { simulation }}{\longrightarrow} \mathrm{N} \text {. }
$$

Although MD simulations of a spin-labeled macromolecule are expected to offer insight into the detailed dynamics of the spin label and its environment, there are important shortcomings to such an approach. In particular, extremely long trajectories are needed to sample the global tumbling of the macromolecule in solution. Without proper sampling of this relatively simple motion, the MD trajectories will not reflect the experimental situation realistically and cw-ESR spectra simulated from them will fail to reproduce the observed spectra. Thus, for the quantitative comparison of simulated and recorded spectra, it becomes necessary to be able to directly account for rotational diffusion by relying on a stochastic model. This can be achieved by modeling the dynamics of the coordinate frame $\mathrm{N}$ with respect to the macromolecule with atomistic MD simulations, while generating the dynamics of M relative to $L$ using a stochastic model of isotropic or anisotropic rotational diffusion: ${ }^{3}$

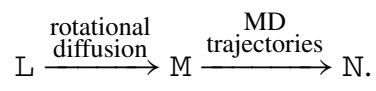

\footnotetext{
${ }^{3}$ Splitting the molecular motion according to (10.7) assumes that the overall molecular tumbling and the motion of the spin label with respect to the global molecular frame are independent [23]. Clearly, this approximation may break in some cases, e.g., when an internal structural rearrangement changes the overall structure-and hence the rotational diffusion tensor-of the whole molecule. Nevertheless, in many instances with spin-labeled biomacromolecules the approximation of decoupled global and internal motions is well justified.
}

$\mathrm{Cw}-\mathrm{ESR}$ spectra simulated from such a combination of stochastic rotational diffusion and MD trajectories were presented in Refs. [47, 49]. Essentially the same approach has been used by DeSensi et al. [12].

Even as MD simulations become more and more routine, the demand on the number and duration of the MD trajectories may become rapidly wasteful and inefficient when the purpose is to insert these into the model (10.7). In particular, the spin dynamics must be propagated over multiple molecular correlation times to explore all the possible orientations and yield a converged ESR spectrum. For this reason, it is important to develop alternative stochastic models able to provide a realistic "mimic" of the long-time dynamics of the spin label relative to the protein. When this dynamics is dominated by rotameric isomerization, the intermittent nature of the transitions between the various rotamers suggests that a Markov state model (MSM) shall provide an ideal framework to encode the internal spin-label dynamics available from MD simulations. Once its parameters have been properly estimated, the so-constructed MSM allows for the generation of computationally inexpensive and arbitrarily long stochastic trajectories. Combining the MSM dynamics with a diffusive model of the tumbling of the protein, cw-ESR spectra can be simulated in time domain according to the scheme:

$$
\mathrm{L} \stackrel{\substack{\text { rotational } \\
\text { diffusion }}}{\longrightarrow} \mathrm{M} \stackrel{\begin{array}{c}
\text { MSM } \\
\text { trajectories }
\end{array}}{\longrightarrow} \mathrm{N} \text {. }
$$

Such simulations were performed in Refs. [49, 50].

\subsubsection{Isotropic and Anisotropic Rotational Diffusion}

To illustrate the impact of rotational diffusion on cw-ESR spectra, we consider the motional model

$$
\mathrm{L} \stackrel{\substack{\text { rotational } \\ \text { diffusion }}}{\longrightarrow} \mathrm{M} \stackrel{\text { fixed }}{\longrightarrow} \mathrm{N} \text {, }
$$

which describes a spin label rigidly tethered to a biological macromolecule tumbling in solution. For concreteness, let us take a double-helical BDNA consisting of 20 base pairs (Fig. 10.2, left), 

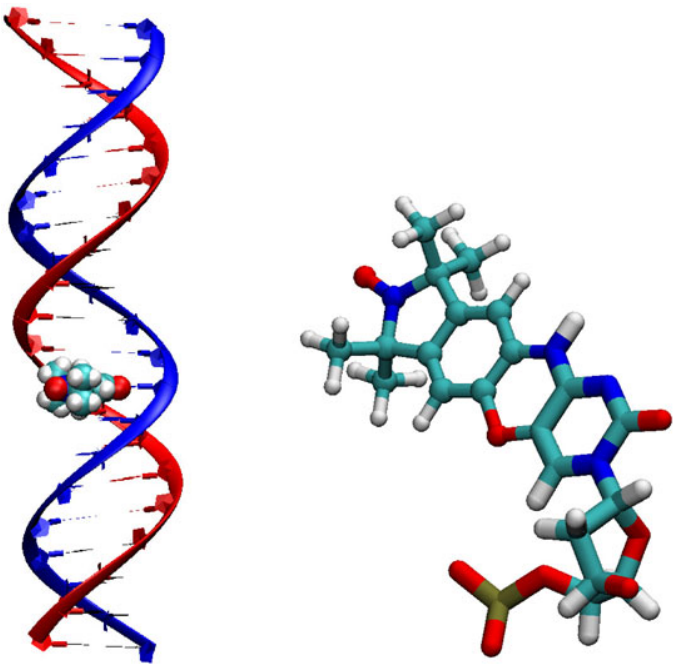

Fig. 10.2 Left: Double-helical B-DNA containing 20 base pairs and labeled with a single nitroxide spin label. Right: Nitroxide spin label (balls and thin sticks) covalently attached to the cytosine nucleotide (thick sticks) of DNA or RNA [9]

one cytosine base of which is labeled with a nitroxide spin label (Fig. 10.2, right) [9]. From the labeling geometry, the $z$ axis of the nitroxide frame (defined in Fig. 10.1) is seen to be perpendicular to the plane of the base and collinear with the helix axis of the DNA. Choosing the $z$ axis of the macromolecular coordinate system as the helix axis, and taking into account the symmetry of the double helix under rotation about this axis, we can take the two coordinate systems $\mathrm{M}$ and $\mathrm{N}$ to be identical.

In general, a $3 \times 3$ diffusion tensor needs to be specified for the tumbling of the molecular frame $\mathrm{M}$ with respect to the laboratory system of axes L. With the above choice of the coordinate axes on the macromolecule the diffusion tensor is expected to be diagonal and of the form $D=\operatorname{diag}\left(D_{\perp}, D_{\perp}, D_{\|}\right)$, where $D_{\|}$and $D_{\perp}$ are the diffusion coefficients for rotation about directions, respectively, parallel and perpendicular to the helix axis. Since the length of the double helix $(\approx 70 \AA)$ is several times larger than its diameter ( $\approx 20 \AA$ ), we expect to have $D_{\|}>D_{\perp}$.

Simulations of cw-ESR spectra at two different magnetic fields ( $B=0.35$ and $B=3.4$ Tesla) for the spin-labeled B-DNA tumbling in solu-
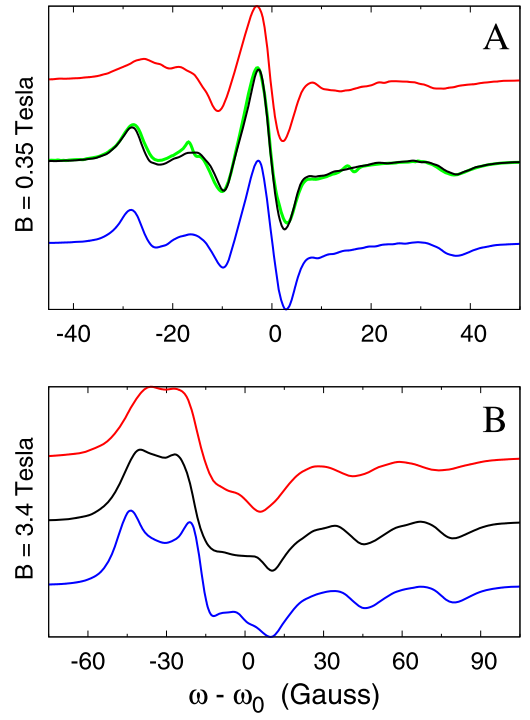

Fig. 10.3 Experimental X-band cw-ESR spectrum (green) and spectra calculated at two different magnetic fields (A) $B=0.35 \mathrm{~T}$ and (B) $B=3.4 \mathrm{~T}$. At each field, three different tumbling rates with the following rotational diffusion tensors are compared: $D=\operatorname{diag}(10,10,10) \times$ $10^{6} \mathrm{~s}^{-1}$ (blue), $D=\operatorname{diag}(10,10,40) \times 10^{6} \mathrm{~s}^{-1}$ (black), and $D=\operatorname{diag}(25,25,40) \times 10^{6} \mathrm{~s}^{-1}($ red $)$. For visual purposes the three spectra are systematically shifted in the vertical direction, which corresponds to the spectral intensity (in arbitrary units)

tion are presented in Fig. 10.3. (For further details about the geometry and the simulation parameters the reader is referred to Ref. [51].) The bottom spectrum (blue), simulated using $D=\operatorname{diag}(10,10,10) \times 10^{6} \mathrm{rad}^{2} / \mathrm{s}$, corresponds to isotropic rotational diffusion expected from a spherical macromolecule. The spectrum in the middle (black), simulated using $D=\operatorname{diag}(10,10$, 40) $\times 10^{6} \mathrm{rad}^{2} / \mathrm{s}$, takes into account the faster diffusion of the elongated DNA molecule about its helix axes. In fact, it compares very well with the experimental spectrum (green) on top of which it is overlaid. Notice, however, that the two spectra (black and blue) at $B=0.35 \mathrm{~T}$ - the magnetic field most commonly used in studies of biomacromolecules - are indistinguishable in these two cases. For the spin labeling geometry considered in this example, cw-ESR experiments at the higher field of $B=3.4 \mathrm{~T}$ are necessary to pick up the elongated shape of the molecule. The (red) spectrum at the top in Figs. 10.3A 

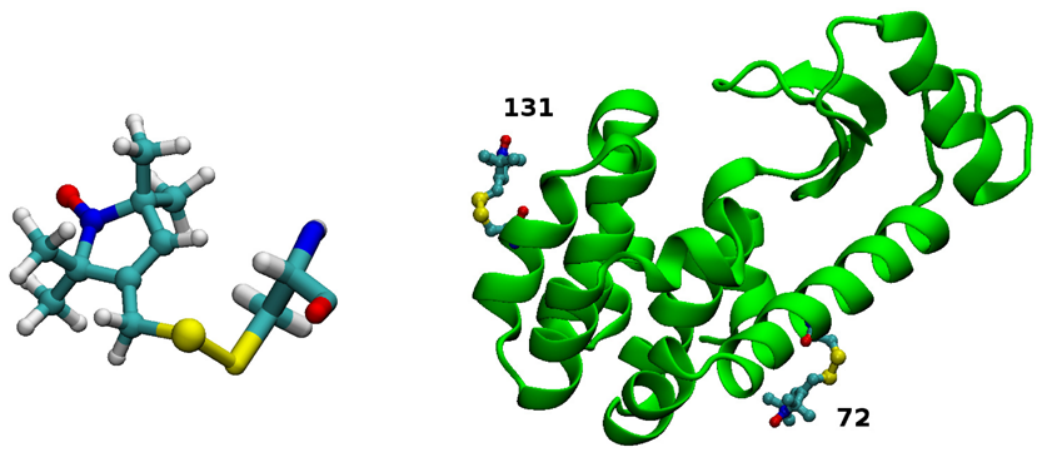

Fig. 10.4 Left: Nitroxide spin label R1 (balls and thin sticks) covalently bonded to the cysteine amino acid (thick sticks) of a protein. Right: T4 Lysozyme (T4L) labeled with $\mathrm{R} 1$ at positions 72 and 131 . The former is located on a long $\alpha$-helix, whereas the latter is on a short $\alpha$-helix. At both sites the spin labels are at the surface of the protein nicely exposed to the solvent, i.e., solvent-exposed helix surface (SEHS) spin labels. In experiments the labels are present one at a time and $\mathrm{B}$, simulated using $D=\operatorname{diag}(25,25,40) \times$ $10^{6} \mathrm{rad}^{2} / \mathrm{s}$, is intended to illustrate the effect of shortening the length of the DNA double helix. Keeping the diffusion coefficient for rotation about the helix axis the same as in the (black) spectrum in the middle, we have increased the rate of diffusion about the perpendicular axis from 10 to $25 \times 10^{6} \mathrm{rad}^{2} / \mathrm{s}$. In this case, the spectra at both fields are sufficiently different from the spectra simulated using $D=\operatorname{diag}(10,10,40) \times$ $10^{6} \mathrm{rad}^{2} / \mathrm{s}$ (black in Fig. 10.3), showing the sensitivity of the experiments to the length of the DNA double helix.

The simulations in Fig. 10.3 rest on the assumption that the only motion experienced by the spin label is anisotropic rotational diffusion. In reality, other motions-like the libration of the base to which the spin label is covalently attached-are expected to take place in addition to the global molecular tumbling. However, it should be clear from the presented evidence that the internal motions can be unambiguously inferred from the experimental spectra only if the effect of the global motion is carefully accounted for along the lines illustrated in Fig. 10.3.

\subsection{MSM of Spin-Label Dynamics}

In this section, we observe that the internal dynamics of a solvent-exposed spin label on the surface of a protein is dominated by the isomeriza- tion of its linker (Sect. 10.3.1). Such motion is perfectly suited for modeling by MSMs that can be subsequently used to simulate cw-ESR spectra according to the model (10.8). The construction of MSMs of the spin-label dynamics from MD trajectories is illustrated in Sect. 10.3.2 for two spin labels at solvent-exposed positions on the protein T4 Lysozyme. Multifrequency spectra for these two positions will be considered in Sect. 10.5.3.

\subsubsection{Side Chain Isomerization as Intermittent Dynamics}

The covalent attachment of the spin label in Fig. 10.2 to the cytosine base of DNA lacks any rotatable bonds. Hence, its internal dynamics is expected to be tightly coupled to the internal dynamics of the entire DNA fragment. The situation is different for ESR studies of proteins, in which the spin label referred to as R1 is most commonly used $[10,16]$. This nitroxide spin label is covalently bonded to the side chain of the amino acid cysteine through a linker consisting of five sequential chemical bonds (Fig. 10.4, left). In principle, rotations around each one of these bonds are sterically permitted, which should allow for rich internal spin label dynamics largely independent from the dynamics of the protein backbone. 

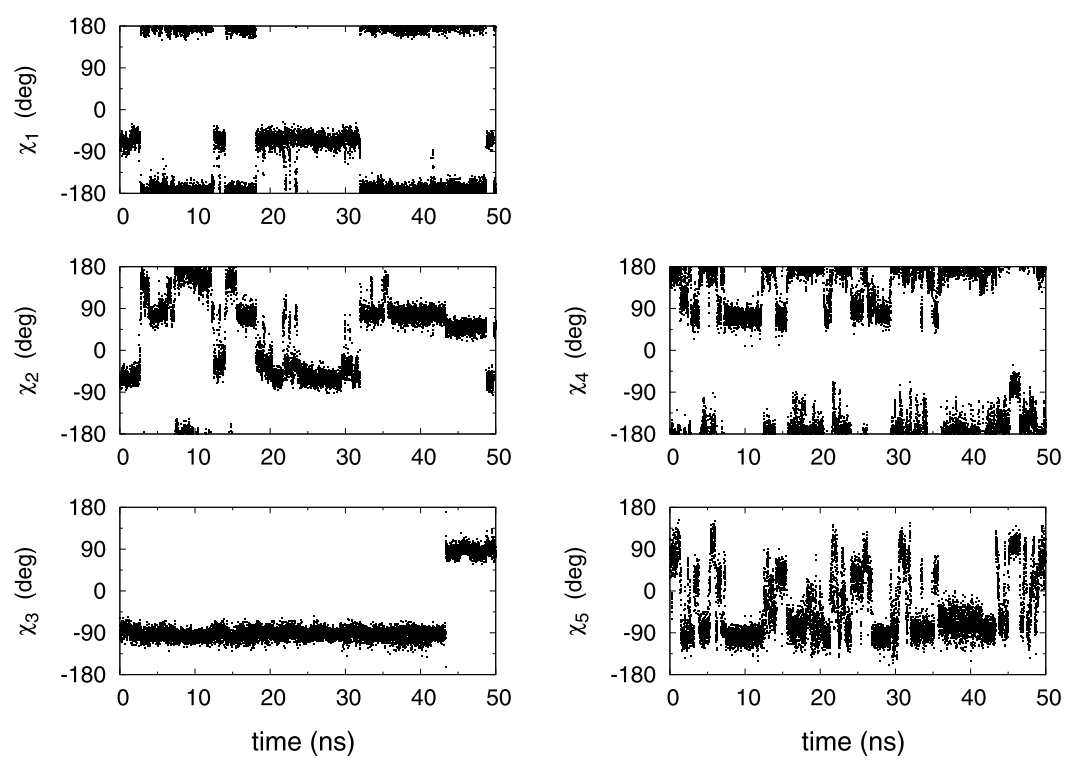

Fig. 10.5 Isomerization dynamics of the nitroxide spin label R1 at position 72 on T4 Lysozyme

Because of the additional complexity introduced by the internal freedom of R1 and other similar spin labels attached to proteins, extensive efforts have been dedicated to elucidate the microscopic factors affecting their conformation and dynamics. Particularly informative are the experimental studies with the well-characterized protein T4 Lysozyme (T4L). A wealth of results, ranging from X-ray crystallography of spinlabeled T4L [18, 21, 31], to X-band [11, 35, 36] or multifrequency $[33,56] \mathrm{cw}-E S R$ experiments, are now available. From those studies, two positions-72 and 131-situated in the middle of, respectively, a long and a short helix, have emerged as prototypical solvent-exposed helix surface (SEHS) sites (Fig. 10.4, right). The dynamics of $\mathrm{R} 1$ at these positions has been rationalized in terms of the " $\chi_{4} / \chi_{5}$ model" for SEHS sites $[10,11]$, which assumes that the internal motion of R1 is largely limited to rotations about the last two dihedrals of the side chain. According to this model, the remaining dihedrals are effectively "locked": the $\chi_{3}$ disulfide torsion is opposed by a large energy barrier [25], while the $\chi_{1}$ and $\chi_{2}$ torsions of the cysteine side chain are hindered by the formation of a hydrogen bond between the sulfur atom of R1 and the backbone amide [36] or $\mathrm{C}_{\alpha}$ [10]. Such sulphur-backbone contacts are indeed observed in a number of $\mathrm{X}$ ray crystal structures of T4L with spin labels $[18,21,31]$, in support for the $\chi_{4} / \chi_{5}$ model. Furthermore, the $\chi_{4} / \chi_{5}$ model offers an atomistic rationalization of the fitting parameters of the diffusional models MOMD and SRLS, which can produce simulated spectra in quantitative agreement with experiments [56].

According to the current understanding, 72R1 and 131R1 in T4L are believed to exemplify the internal R1 dynamics at SEHS sites in maximum isolation. The differences in the X-band cw-ESR spectra at these two positions are thought to reflect the effect of backbone motion on the mobility of the spin label side chain [11]. Nevertheless, a number of issues remain. For instance, the spin label is partly disordered and unresolved in several X-ray structures [18], suggesting that multiple conformations are energetically accessible. Therefore, in spite of the large amount of data available, a definitive characterization of the spin label dynamics at SEHS sites at the atomic level has not been achieved.

The values of the spin label dihedral angles during an atomistic simulation of T4L labeled at position 72 are shown in Fig. 10.5. The 
Table 10.1 Information about the two sets of MD simulations of spin-labeled T4 Lysozyme

\begin{tabular}{lll}
\hline & $72 \mathrm{R} 1(\mathrm{set} 1)$ & $131 \mathrm{R} 1(\mathrm{set} 2)$ \\
\hline Number of independent trajectories & $18\left(\chi_{1}, \chi_{2}, \chi_{3}\right)^{\mathrm{a}}$ & $54\left(\chi_{1}, \chi_{2}, \chi_{3}, \chi_{4}\right)^{\mathrm{a}}$ \\
\hline Duration of single trajectory & $32.3 \mathrm{~ns}$ & $12.7 \mathrm{~ns}$ \\
\hline Total simulation time (analyzed) $^{\mathrm{b}}$ & $581(563) \mathrm{ns}$ & $686(632) \mathrm{ns}$ \\
\hline
\end{tabular}

${ }^{a}$ In each independent trajectory of a given set the spin label was initialized to be in a different rotameric state by restraining the dihedral angles given in the parenthesis to their canonical values. The number of different rotamers was determined using the multiplicity of the dihedral angles, $\chi_{1}: 3, \chi_{2}: 3, \chi_{3}: 2, \chi_{4}: 3$

${ }^{\mathrm{b}}$ The first $1 \mathrm{~ns}$ of every trajectory was treated as equilibration period and not analyzed

time traces of these angles do seem to undergo jump like dynamics between different discrete states, exemplifying intermittent internal dynamics. Thus, the transitions between the rotameric conformations of the spin label side chain should be amenable to modeling by MSMs.

Before embarking on a rigorous MSM modeling, a few observations about the internal isomerization dynamics of the protein spin label can be made on the basis of the time traces in Fig. 10.5. First, the lifetimes of the states corresponding to the different values that the five torsions preferentially adopt show great variation. At one extreme is the disulfide dihedral angle $\chi_{3}$, which has undergone a single transition from $\chi_{3} \approx-90^{\circ}$ to $\chi_{3} \approx+90^{\circ}$ during the entire simulation of $50 \mathrm{~ns}$. At the other extreme is the dihedral angle closest to the nitroxide ring, $\chi_{5}$, which has moved several times between the values $\chi_{5} \approx-90^{\circ}, \chi_{5} \approx+90^{\circ}$ and $\chi_{5} \approx 0^{\circ}$. Second, $\chi_{1}$ and $\chi_{2}$-the dihedral angles closest to the protein backbone-are seen to undergo a transition every 5 to $10 \mathrm{~ns}$. As a result, $\chi_{2}$ has visited the three canonical values of $\pm 60^{\circ}$ and $180^{\circ}$, even if only a few times. Similarly, $\chi_{1}$ has exchanged between two of these canonical values a few times. ${ }^{4}$ Third, in many of the transitions $\chi_{1}$ and $\chi_{2}$ seem to flip simultaneously in a concerted manner. Occasionally, all the four dihedral angles, with the exception of $\chi_{3}$, are seen to undergo concerted transitions. Furthermore, the rate of isomerization of a given

\footnotetext{
${ }^{4}$ In protein crystal structures the side chain of cysteine is very rarely seen to adopt a conformation with $\chi_{1} \approx+60^{\circ}$ when located on $\alpha$ helices since this places the cysteine sulfur atom in unfavorable steric contact with the backbone atoms of the helix.
}

dihedral appears to depend on the values adopted by all the other dihedral angles. (This is perhaps most clearly seen for $\chi_{5}$.) Hence, it is not justified to assume that the dynamics of the torsion angles is independent [54]. Instead, the conformation of the entire spin label side chain has to be considered when trying to identify the states of the intermittent motion and the rates of exchange between them. In the light of these observations we now turn to the systematic construction of MSMs for the dynamics of R1 at positions 72 and 131 in $\mathrm{T} 4 \mathrm{~L}$.

\subsubsection{MSM from MD Trajectories}

Extensive all-atom MD simulations of fullysolvated spin-labeled $\mathrm{T} 4 \mathrm{~L}$ were performed for the two SEHS positions of interest-72 and 131with the purpose of mapping the R1 isomerization dynamics from the MD trajectories to MSMs. To enhance the sampling of the possible spin-label conformations several independent trajectories were generated starting from different $\mathrm{R} 1$ conformations. Information about the number and duration of the trajectories is summarized in Table 10.1.

To proceed with building MSMs of the spinlabel dynamics relative to the protein from the MD simulations, a set of observables, called order parameters, has to be selected among the large collection of variables contained in the trajectories. Here, we assume that the dihedral angles of the spin label side chain constitute an adequate set of order parameters - a natural choice based on physical insight about the system. Then, 
the five-dimensional space of the order parameters is divided into 120 regions $^{5}$ (microstates) using K-means clustering [24]. At this point, it is hoped that if the microstates are chosen to be narrow enough, such that intrastate relaxation is fast, the kinetics of jumping out of a microstate will be approximately Markovian.

More formally, let $X(t)$ be a random variable indicating the state of an $N$-state Markov chain model at time $t$. The probabilities $p_{i}(t)=$ $\mathbb{P}\{X(t)=i\}$, to observe the chain in state $i$ at time $t$, form a (row) vector $\langle p(t)|=\left[p_{i}(t)\right]$, whose evolution is governed by the Master equation

$$
\dot{p}_{j}(t)=\sum_{i=1}^{N} p_{i}(t) K_{i j} .
$$

Here, a derivative with respect to time has been denoted with a dot. The matrix $K=\left[K_{i j}\right]$ is referred to as the "rate matrix". Its off-diagonal entries are larger or equal to zero. For a conservative process, its diagonal elements are negative and given as $K_{i i}=-\sum_{j \neq i} K_{i j}$. They are directly related to the lifetime [39]

$$
v_{i}=-1 / K_{i i}
$$

of each state. The stationary probability distribution of the chain $\langle\pi|$, is the left eigenvector of $K$ with eigenvalue zero, i.e., $\langle\pi| K=0$. A system in thermal equilibrium satisfies the detailed balance condition $\pi_{i} K_{i j}=\pi_{j} K_{j i}$, which implies that $K$ can be transformed to a symmetric form by a similarity transformation with the diagonal matrix $D=\left[\sqrt{\pi_{i}} \delta_{i j}\right]$. Thus all the eigenvalues of $K$ are real. When written as $-1 / \tau_{i}$, the nonzero eigenvalues give the relaxation time scales $\tau_{i}$ of the stochastic dynamics generated by $K$ (cf. Eq. (10.12)). Note that, in general, $\tau_{i} \neq v_{i}$.

If the observed time series were generated from a continuous-time Markov chain, one could easily estimate the rate matrix by counting the number of $i \rightarrow j$ jumps and the total time spent

\footnotetext{
${ }^{5}$ The observation that, considering the multiplicity of its dihedral angles, the R1 side chain can adopt 108 different rotameric states motivated the choice of number of microstates
}

in state $i$, i.e., lifetime $v_{i}$. This is not possible when the trajectories of the order parameters are coming from MD simulations, since the shorttime dynamics of the order parameters are not necessarily Markovian. For instance, the timeseries of the spin-label torsion angles in Fig. 10.5 contain "spurious" transitions back and forth between states $i$ and $j$ before a "real" transition occurs. This would lead to an unreliable estimate of $K$ from the MD trajectories. This problem is partially alleviated by observing the system only at instances separated by a long enough time interval $\tau$-referred to as lag time — such that the dynamics is more likely to appear memoryless from one observation to the next. Such an approach, however, leads to a discretization of the time axis, thus deviating from the continuous-time Markov chain model (10.10). By counting the number of times the chain in state $i$ goes to state $j$ after time $\tau$, a transition probability matrix, $T(\tau)$, with matrix elements $T_{i j}(\tau)=\mathbb{P}\left\{X_{t+\tau}=j \mid X_{t}=i\right\}$ can be estimated. This matrix determines the evolution of the state probabilities for times spaced by $\tau:\langle p(t+\tau)|=\langle p(t)| T(\tau)$.

To further reduce the miscounting of very short-lived excursions in the values of the torsion angles as genuine transition events between distinct conformational states, the time series of the spin label dihedral angles were analyzed with a hidden Markov model. The latter allows for the observed dihedral angle values to be dispersed about the values defining the state of the MSM according to a distribution with a mean and a standard deviation. In this sense, what is analyzed are the values of the dihedral angles "emitted" from a rotameric state that is not directly accessible (i.e., hidden). The mean values of the observed torsion angles in each state as well as the state-to-state jump probabilities were inferred in an iterative manner using the Viterbi algorithm [42]. Detailed description of the followed procedure is available in Ref. [49].

Ideally, if the modeled process is indeed Markovian, the transition matrices estimated using different lag times should be consistent in the sense that $T\left(\tau_{1}\right) T\left(\tau_{2}\right)=T\left(\tau_{1}+\tau_{2}\right)$. If the underlying process being observed at discrete instances in time is in fact a continuous-time Markov chain 
Fig. 10.6 Relaxation time scales calculated according to $(10.12)$ using the eigenvalues of $T(\tau)$ estimated from the data at various lag times $\tau$. The thick black curves correspond to $2 \tau . \tau_{i}$ 's that fall under these curves are essentially zero and are poorly estimated

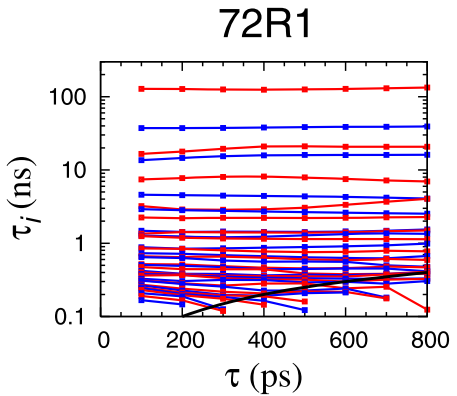

with rate matrix $K$, the relation $T(\tau)=\exp (\tau K)$ should hold. This implies that the eigenvalues $\lambda_{i}^{T}(\tau)$ of $T(\tau)$ are related to the eigenvalues $\lambda_{i}^{K}$ of $K$ through $\lambda_{i}^{T}(\tau)=\exp \left(\tau \lambda_{i}^{K}\right)$. Therefore, the relaxation time scales of the MSM are

$$
\tau_{i}=-\frac{1}{\lambda_{i}^{K}}=-\frac{\tau}{\ln \lambda_{i}^{T}(\tau)} .
$$

Equation (10.12) is a necessary, although not sufficient, condition for the matrices $T(\tau)$ estimated using different lag times $\tau$ to describe a genuinely Markovian process. If the relaxation time scales $\tau_{i}$ calculated from the eigenvalues of such $T(\tau)$ are not independent of $\tau$, it is certain that the process is not Markovian. To assess the Markovian nature of the estimated process corresponding to the isomerization of the spin label R1 at positions 72 and 131, the analysis of the dihedral time series using the Viterbi algorithm was repeated for several different lag times $\tau$, ranging from 100 to $800 \mathrm{ps.} \mathrm{The} \mathrm{relax-}$ ation time scales obtained from the estimated microstate transition matrices according to (10.12) are shown in Fig. 10.6. The fact that the lines are approximately horizontal (i.e., independent of the lag time) indicates that, on time scales larger than the lag time, the time series for dihedral dynamics of both 72R1 and 131R1 are faithfully modeled by MSMs of jumps between 120 discrete states. Further analysis is limited to the transition probability matrices for the shortest lag time, $\tau=100$ ps.

The choice of microstates based on K-means clustering is purely geometrical. Using the microstate transition probability matrix the microstates can be lumped into several groups of kinetic significance (macrostates). The resulting macrostates are intended to correspond to the rarely exchanging, metastable conformations of the spin label, and in the end, it is the Markovian kinetics among the macrostates that constitutes a model of the slow spin-label dynamics relative to the protein. The degree of lumping, which determines the final number of macrostates, is decided on the basis of the desired temporal resolution (which is related to the width of the cw-ESR spectrum, as discussed in Sect. 10.5.2).

The final MSMs constructed for $72 \mathrm{R} 1$ and 131R 1 on T4L contained 37 and 38 (macro)states, respectively (Table 10.2). It is important to note that a critical ingredient of the MSMs at this stage is the usage of pre-averaged magnetic tensors of the spin labels associated with the Markovian macrostates (see Sects. 10.5.1 and 10.5.2). At both sites, the slowest relaxation times $\left(\tau_{1} \gtrsim\right.$ $100 \mathrm{~ns}$ ) are related to transitions of the disulfide torsion angle between its two stable conformations $\chi_{3} \approx-90^{\circ}(\mathrm{m})$ and $\chi_{3} \approx+90^{\circ}(\mathrm{p})$. The exact numerical values of $\tau_{1}$, as well as the relative populations of the $m$ and $p$ conformations are not expected to be accurately estimated by the constructed MSM due to the small number of such transitions observed in the free simulations (Table 10.2). To determine accurately the m:p ratio, the free energy difference between two Markovian states on the opposite side of the $\chi_{3}$ torsion was calculated using umbrella sampling simulations [45]. This resulted in $27 \% \mathrm{~m}-73 \%$ $\mathrm{p}$ for $72 \mathrm{R} 1$, and $55 \% \mathrm{~m}-45 \% \mathrm{p}$ for $131 \mathrm{R} 1$ (Table 10.2) [50].

The spin-label conformations of the most populated five states of the MSMs of 72R1 and 131R1 are presented in Fig. 10.7. In spite of the 
Table 10.2 Number of transitions between the $m$ and $p$ conformations observed in all the MD trajectories and the number of Markov states assigned to those conformations

\begin{tabular}{|c|c|c|c|c|c|c|}
\hline \multirow[t]{2}{*}{ site } & \multicolumn{2}{|c|}{ number of transitions } & \multicolumn{3}{|c|}{ number of states } & \multirow[t]{2}{*}{$\mathrm{m}: \mathrm{p}^{\mathrm{a}}$} \\
\hline & $\mathrm{m} \rightarrow \mathrm{p}$ & $\mathrm{p} \rightarrow \mathrm{m}$ & $\mathrm{m}$ & $\mathrm{p}$ & total & \\
\hline 72R1 & 2 & 1 & 18 & 19 & 37 & $27: 73$ \\
\hline 131R1 & 1 & 1 & 19 & 19 & 38 & 55:45 \\
\hline
\end{tabular}

${ }^{\mathrm{a}}$ The m:p ratio $(\%)$ is determined from independent restrained simulations
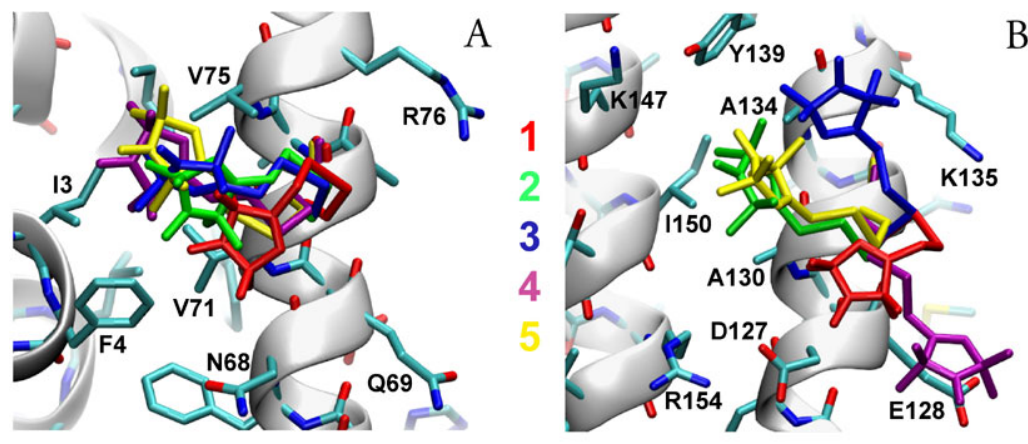

C

MNIFEMLRID EGLRLKIYKD TEGYYTIGIG HLLTKSPSLN AAKSELDKAI GRNTNGVITK DEAEKLFNQD VCAAVRGILR NAKLKPVYDS LDAVRRAALI NMVFQMGETG VAGFTNSLRM $\uparrow * \uparrow$ LQQKRWDEAA CNLAKSRWYN QTPNRAKRVI TTFRTGTWDA YKNL

Fig. 10.7 The most populated five conformations of 72R1 (A), and 131R1 (B) are colored as indicated. The rotameric states of the neighboring side chains when interacting with $\mathrm{R} 1$ are not necessarily the same as the ones shown here. (C) The amino acid sequence of T4L. Stars indicate the spin labeled sites and arrows point between the $i \pm 3$ and $i \pm 4$ positions. Residues with which R1 interacts are colored red and blue for 72 and 131, respectively variation of $\chi_{4}$ and $\chi_{5}$, as well as $\chi_{1}, \chi_{2}$, and $\chi_{3}$, across the most populated states of $72 \mathrm{R} 1$, the nitroxide ring appears to be rather well localized (Fig. 10.7A). In contrast, the conformations corresponding to the first five most populated states of 131R1 are dispersed (Fig. 10.7B). In the simulations the spin label adopts a multiplicity of conformations, many of which tend to interconvert on time scales relevant for cw-ESR experiments. When the exchanges are slow, the states appear to be stabilized by a range of polar and nonpolar interactions with neighboring amino acids (Fig. 10.7C) frequently involving the nitroxide ring.

The MSMs of spin-label dynamics at positions 72 and 131 in T4L constructed from the MD simulations can be used in the model (10.8) to simulate cw-ESR spectra. The results of such simulations are compared with experiments in Sect. 10.5.3. In the next section we describe the procedure for simulating cw-ESR spectra from trajectories of the spin-label dynamics.

\subsection{Numerical Simulation of cw-ESR Spectra}

$\mathrm{Cw}-\mathrm{ESR}$ experiments are performed under the presence of a strong constant magnetic field and a weak microwave (mw) magnetic field applied continuously in a direction perpendicular to the constant field. Subjecting the sample to these external magnetic fields leads to detectable bulk magnetization. Cw-ESR experiments measure the component of the magnetization perpendicular to the constant magnetic field-transverse component; the longitudinal component along 
the direction of the constant magnetic field is not detected. For the sufficiently weak mw fields typically employed, the cw spectrum is identical to the Fourier transform of a free induction decay (FID) [1], where FID refers to the decay of the transverse magnetization in the absence of any mw field. In FID the electron spins are first flipped to the transverse plane using a ninetydegree mw pulse after which the oscillating field is switched off. Hence, at the beginning of the decay, the longitudinal magnetization is equal to zero while the transverse magnetization can be taken to be equal to one. During an FID the spins evolve only under the action of the constant magnetic field and the observed decay of the transverse magnetization results from the decoherence of the spins. In magnetic resonance the time scale of this decay is known as $T_{2}$. In contrast, the time scale on which the longitudinal magnetization builds up is referred to as $T_{1}$. At sufficiently strong magnetic fields and for motionally broadened cw-ESR spectra, which are our main interest, $T_{2}$ is much shorter than $T_{1}$ (e.g., nanoseconds versus microseconds). Therefore, it is safe to assume that by the time the transverse magnetization has decayed to zero the longitudinal magnetization has remained at its initial value of zero. This constitutes the high-field approximation whose implications will become apparent below (see Sect. 10.4.1).

Because there is no mw field during the FID evolution, in numerical work aiming to calculate cw-ESR spectra it is preferable to simulate the FID and compute its Fourier transform. With $\mathscr{M}_{+}(t)$ denoting the bulk transverse magnetization after a ninety-degree pulse, the spectrum is the (one-sided) Fourier transform

$$
\mathscr{S}(\omega)=\int_{0}^{\infty} \mathscr{M}_{+}(t) \mathrm{e}^{-\mathrm{i} \omega t} \mathrm{~d} t .
$$

Due to the way a cw experiment is actually performed-which is different than recording an FID - the spectrum is in fact the derivative of $\mathscr{S}(\omega)$ with respect to $\omega$. Differentiating (10.13), a derivative-mode cw-ESR spectrum is readily found to be

$$
\frac{\mathrm{d} \mathscr{S}(\omega)}{\mathrm{d} \omega}=-\mathrm{i} \int_{0}^{\infty} t \mathscr{M}_{+}(t) \mathrm{e}^{-\mathrm{i} \omega t} \mathrm{~d} t .
$$

Using two channels both the real and imaginary parts of this spectrum can be recorded. In practice, however, only the real part is reported. All the calculated cw-ESR spectra shown in this chapter correspond to the real part of (10.14), obtained by taking the discrete Fourier transform of $t \mathscr{M}_{+}(t)$ numerically. Because it follows the decay of $\mathscr{M}_{+}(t)$ in time, the presented approach constitutes a simulation in time domain (in contrast to the frequency-domain methodology based on the SLE).

\subsubsection{The Nitroxide Density Matrix}

To calculate the macroscopic transverse magnetization appearing in (10.14), the microscopic magnetizations, $M_{+}(t)$, from all the possible random trajectories that a nitroxide spin label in the solution may undergo need to be averaged. Denoting this ensemble averaging with angular brackets we have

$$
\mathscr{M}_{+}(t)=\left\langle M_{+}(t)\right\rangle \text {. }
$$

Assuming a dilute solution of non-interacting free radicals, the state of each electron-nuclear spin system on one nitroxide can be described by a density operator. With $\rho(t)$ denoting the density matrix associated with one stochastic trajectory of an individual spin label, microscopic transverse and longitudinal magnetizations can be calculated as follows: ${ }^{6}$

$$
\begin{aligned}
& M_{+}(t)=\operatorname{Tr}\left\{\rho(t) \hat{S}_{+}\right\} \quad \text { and } \\
& M_{z}(t)=\operatorname{Tr}\left\{\rho(t) \hat{S}_{z}\right\} .
\end{aligned}
$$

\footnotetext{
${ }^{6}$ Since the absolute value of the cw-ESR measurement depends on instrumental factors and is not relevant for our purposes, proportionality constants relating the magnetizations and the respective spin operators have been neglected in (10.16).
} 
In these expressions, $\hat{S}_{+}$and $\hat{S}_{z}$ are spin-1/2 operators ${ }^{7}$ and $\mathrm{Tr}$ denotes a trace. The density operator lives in the outer product of the electron and nuclear spin Hilbert spaces. For easier access to the microscopic magnetizations in (10.16) it can be written as a sum of Kronecker products between the spin-1/2 matrices and matrices $\rho_{\kappa}$ with the dimensionality of the nuclear-spin Hilbert space: ${ }^{8}$

$$
\begin{aligned}
\rho(t)= & \rho_{+}(t) \hat{S}_{-}+\rho_{-}(t) \hat{S}_{+}+2 \rho_{z}(t) \hat{S}_{z} \\
& +\rho_{0}(t) \hat{S}_{0} \\
= & {\left[\begin{array}{cc}
\rho_{0}+\rho_{z} & \rho_{-} \\
\rho_{+} & \rho_{0}-\rho_{z}
\end{array}\right] . }
\end{aligned}
$$

Thus, $\rho$ is represented by a $6 \times 6$ matrix (for ${ }^{14} \mathrm{~N}$ ) or a $4 \times 4$ matrix (for ${ }^{15} \mathrm{~N}$ ). Using (10.17) in (10.16) it is straightforward to deduce that

$$
\begin{aligned}
M_{+}(t) & =\operatorname{Tr}\left\{\rho_{+}(t)\right\} \quad \text { and } \\
M_{z}(t) & =\operatorname{Tr}\left\{\rho_{z}(t)\right\},
\end{aligned}
$$

where the trace in these expressions is only over the nuclear spin degrees of freedom.

To calculate the microscopic magnetization $M_{+}(t)$ along a given dynamical trajectory we need to be able to follow numerically the evolution of the density matrix starting from the appropriate initial conditions. To this end, we recall that the density matrix evolves according to the Liouville-von Neumann equation

$$
\dot{\rho}(t)=-\mathrm{i}[\hat{H}(t), \rho(t)]
$$

${ }^{7}$ These are

$$
\begin{array}{ll}
\hat{S}_{0}=\left[\begin{array}{ll}
1 & 0 \\
0 & 1
\end{array}\right], & \hat{S}_{+}=\left[\begin{array}{ll}
0 & 1 \\
0 & 0
\end{array}\right], \\
\hat{S}_{-}=\left[\begin{array}{ll}
0 & 0 \\
1 & 0
\end{array}\right], & \hat{S}_{z}=\frac{1}{2}\left[\begin{array}{cc}
1 & 0 \\
0 & -1
\end{array}\right] .
\end{array}
$$

${ }^{8}$ In principle, the submatrix $\rho_{0}$ in $(10.17)$ contains the $3 \times 3$ or $2 \times 2$ identity matrix $I_{0}$ along its main diagonal. However, the part proportional to the identity matrix is neither affected by the relaxation or the coherent evolution nor does it affect the evolution of the rest of the density matrix. Hence, $\rho_{0}$ can be taken as traceless. where $\hat{H}(t)$ is the Hamiltonian (10.1) of the spin system (in units of angular frequency) and $[\cdot, \cdot]$ denotes a commutator. The initial conditions for an FID can be obtained from (10.18). These are the identity matrix for $\rho_{+}(0)$ and the zero matrix for $\rho_{z}(0)$.

In analogy to (10.17), the ESR spin Hamiltonian can be written as

$$
\hat{H}(t)=2 H_{z}(t) \hat{S}_{z}+H_{+}(t) \hat{S}_{-}+H_{-}(t) \hat{S}_{+},
$$

where the matrices $H_{\kappa}$ have the dimensionality of the Hilbert space of the nuclear spin. Substituting the expansions (10.17) and (10.20) into the equation of motion (10.19), and equating the coefficients of $\hat{S}_{-}$on both sides of the equality leads to the evolution law

$$
\begin{aligned}
\dot{\rho}_{+}(t)= & \mathrm{i}\left\{H_{z}(t), \rho_{+}(t)\right\}-\mathrm{i}\left\{H_{+}(t), \rho_{z}(t)\right\} \\
& -\mathrm{i}\left[H_{+}(t), \rho_{0}(t)\right],
\end{aligned}
$$

where $\{\cdot, \cdot\}$ denotes an anticommutator. At this point, it is convenient to invoke the high-field approximation, which amounts to assuming that $\rho_{z}$ and $\rho_{0}$ remain zero throughout the times we follow the evolution of $\rho_{+} .{ }^{9}$ As a result, (10.21) simplifies to

$$
\dot{\rho}_{+}(t)=\mathrm{i}\left\{H_{z}(t), \rho_{+}(t)\right\} .
$$

Hence, $\rho_{+}(t)$ is the only part of the full density matrix that needs to be considered and $H_{z}(t)$ is the only part of the Hamiltonian that needs to be calculated at every time step. From (10.1),

$$
H_{z}(t)=\left|\gamma_{\mathrm{e}}\right|\left[B G_{z z}^{\mathrm{L}}(t)+\mathbf{a}^{\mathrm{L}}(t) \cdot \hat{\mathbf{I}}\right] / 2,
$$

where $G_{z z}^{\mathrm{L}}(t)$ is the respective component of the rescaled Zeeman tensor in the laboratory frame, and the components of $\mathbf{a}^{\mathrm{L}}$ are defined in terms of the components of the hyperfine tensor as

$$
a_{i}^{\mathrm{L}}(t) \equiv A_{i z}^{\mathrm{L}}(t) .
$$

\footnotetext{
${ }^{9}$ As mentioned above, the justification lies in the fact that the time scale $T_{1}$-on which $\rho_{z}$ and $\rho_{0}$ build up-depends on motions at the time scale of the Larmor precession and is much longer than the time scale $T_{2}$-on which $\rho_{+}$ decays - dominated by slow motions. The high field approximation automatically excludes the possibility to account for the contribution of $T_{1}$ processes to $T_{2}$ relaxation using Eq. (10.22).
} 
Table 10.3 Magnetic fields, corresponding Larmor frequencies, ${ }^{\mathrm{a}}$ microwave bands, ${ }^{\mathrm{b}}$ and time scales of precession ${ }^{\mathrm{c}}$

\begin{tabular}{lllllll}
\hline$B / \mathrm{T}$ & 0.12 & 0.34 & 1.21 & 3.39 & 6.07 & 9.2 \\
\hline$f_{\mathrm{e}} / \mathrm{GHz}$ & $3.4(\mathrm{~S})$ & $9.5(\mathrm{X})$ & $34(\mathrm{Q})$ & $95(\mathrm{~W})$ & $170(\mathrm{G})$ & $260(\mathrm{~J})$ \\
\hline$\tau_{0} / \mathrm{ps}$ & 47 & 17 & 4.7 & 1.7 & 0.95 & 0.61 \\
\hline
\end{tabular}

${ }^{\mathrm{a}} f_{\mathrm{e}}=\omega_{0} / 2 \pi$

${ }^{\mathrm{b}}$ In parenthesis

${ }^{\mathrm{c}} \tau_{0}=1 / \omega_{0}$

\subsubsection{Propagation of the Quantum Spin System}

Each electron spin in the ensemble undergoes a precession about the applied constant magnetic field $B$ with average angular frequency, known as the Larmor frequency of the electron spin, equal to [32]

$$
\omega_{0} \equiv-\gamma_{\mathrm{e}} B G_{0}
$$

where $G_{0}=\operatorname{Tr}\{\mathbf{G}\} / 3$. Table 10.3 contains the frequencies and time scales of precession for several different magnetic fields of experimental interest. When following the time evolution of the density matrix with the purpose of calculating $M_{+}(t)$, it proves convenient to work in a coordinate system rotating about the laboratory $z$-axis with the electron Larmor frequency. In this rotating frame (denoted with a prime) the operators $\hat{S}_{0}$ and $\hat{S}_{z}$ remain unchanged whereas $\hat{S}_{+}$ and $\hat{S}_{-}$acquire a phase: $\hat{S}_{ \pm}^{\prime}=\hat{S}_{ \pm} \mathrm{e}^{ \pm \mathrm{i} \omega_{0} t}$. Hence, from (10.17), the density matrix in the rotating frame becomes $\rho^{\prime}(t)=\rho_{+}^{\prime}(t) \hat{S}_{-}+\rho_{-}^{\prime}(t) \hat{S}_{+}+$ $2 \rho_{z}(t) \hat{S}_{z}+\rho_{0}(t) \hat{S}_{0}$, where

$$
\rho_{ \pm}(t)=\rho_{ \pm}^{\prime}(t) \mathrm{e}^{ \pm \mathrm{i} \omega_{0} t} .
$$

Using this last relation in (10.18) yields the transverse magnetization ${ }^{10}$

$$
M_{+}(t)=\operatorname{Tr}\left\{\rho_{+}^{\prime}(t)\right\} \mathrm{e}^{\mathrm{i} \omega_{0} t}=M_{+}^{\prime}(t) \mathrm{e}^{\mathrm{i} \omega_{0} t} .
$$

\footnotetext{
${ }^{10}$ The numerical advantages associated with working in the rotating frame are apparent from (10.27), where the transverse magnetization $M_{+}(t)$ consists of a rapidly oscillating "carrier" wave whose amplitude is modulated by the slowly changing "signal" $M_{+}^{\prime}(t)$. Thus, following $M_{+}(t)$ numerically would require an integration time step sufficient to resolve the fast oscillations on the time scale of the Larmor precession (cf. Table 10.3). In contrast, calculating the slowly changing $M_{+}^{\prime}(t)$ numerically allows us to take time steps larger by several orders of magnitude.
}

Substituting (10.27) in the expression for the spectrum (10.13), we find

$$
\mathscr{S}\left(\omega-\omega_{0}\right)=\int_{0}^{\infty}\left\langle M_{+}^{\prime}(t)\right\rangle \mathrm{e}^{-\mathrm{i} \omega t} \mathrm{~d} t .
$$

Hence, taking the Fourier transform of the ensemble-average of the slowly varying $M_{+}^{\prime}(t)$ produces the desired experimental cw-ESR spectrum but with the origin shifted to the Larmor frequency $\omega_{0}$.

Since $M_{+}^{\prime}$ is defined as the trace of $\rho_{+}^{\prime}$ the latter needs to be evolved numerically. From (10.22) and (10.26) it is straightforward to conclude that

$$
\begin{aligned}
\dot{\rho}_{+}^{\prime}(t) & =\mathrm{i}\left\{H_{z}(t), \rho_{+}^{\prime}(t)\right\}-\mathrm{i} \omega_{0} \rho_{+}^{\prime}(t) \\
& =\mathrm{i}\left\{V(t), \rho_{+}^{\prime}(t)\right\},
\end{aligned}
$$

where

$$
\begin{aligned}
V(t) & \equiv H_{z}(t)-\omega_{0} / 2 \\
& =\left|\gamma_{\mathrm{e}}\right|\left[B G_{z z}^{\prime \mathrm{L}}(t)+\mathbf{a}^{\mathrm{L}}(t) \cdot \hat{\mathbf{I}}\right] / 2 .
\end{aligned}
$$

In the last equality of (10.30) we have introduced the traceless coupling tensor $\mathbf{G}^{\prime}$ obtained by subtracting $G_{0}$ from the diagonal entries of the Zeeman tensor $\mathbf{G}: \mathbf{G}^{\prime} \equiv \mathbf{G}-G_{0} \mathbf{E}$.

The numerical evolution of $\rho_{+}^{\prime}(t)$ according to (10.29) over a short time step $\Delta t$ can be achieved as $^{11}$

$$
\rho_{+}^{\prime}(t+\Delta t)=U(t, \Delta t) \rho_{+}^{\prime}(t) U(t, \Delta t)
$$

after introducing the short-time propagator

\footnotetext{
${ }^{11}$ Note that the same propagator matrix acts on both sides of $\rho_{+}^{\prime}$ in this equation, which is different from the propagation of the density matrix $\rho$ in the full Hilbert space. The source of the difference lies in replacing the commutator in (10.19) by an anticommutator in (10.22).
} 


$$
\begin{aligned}
U(t, \Delta t) & \equiv \mathrm{e}^{\mathrm{i} \Delta t V(t)} \\
& =\mathrm{e}^{\mathrm{i} \Delta t\left|\gamma_{\mathrm{e}}\right| B G_{z z}^{\prime \mathrm{L}}(t) / 2} \mathrm{e}^{\mathrm{i} \Delta t\left|\gamma_{\mathrm{e}}\right| \mathbf{a}^{\mathrm{L}}(t) \cdot \hat{\mathbf{I}} / 2},
\end{aligned}
$$

which needs to be calculated from the instantaneous values of the magnetic tensors in the laboratory frame at every integration time step. (How to efficiently compute $U(t, \Delta t)$ was described in Ref. [48].)

The resulting computational framework is summarized by the following steps:

(i) To represent the dynamics of the classical degrees of freedom, a stochastic trajectory combining continuous rotational diffusion together with an MSM according to the model (10.8) is propagated, generating the time series $R^{\mathrm{LN}}(t)=R^{\mathrm{LM}}(t) R^{\mathrm{MN}}(t)$ sampled at the time step $\Delta t$.

(ii) At every time step the instantaneous values of the magnetic tensors in the laboratory frame are calculated according to (10.4) and used to obtain the short-time propagator (10.32). (It should be noted that these correspond to the magnetic tensors averaged over the fast librations of the spin label assigned to a given Markovian macrostate, as described in Sects. 10.5.1 and 10.5.2).

(iii) The QM density matrix, $\rho_{+}(t)$, is evolved along a single stochastic trajectory according to (10.31) and a microscopic magnetization $M_{+}^{\prime}(t)$ is calculated from its trace at every time step $\Delta t$.

(iv) A large number of such trajectory-specific magnetization time series, calculated by generating different realizations of the stochastic trajectories, are generated and added together to obtain the ensembleaveraged macroscopic magnetization in the rotating frame, $\left\langle M_{+}^{\prime}(t)\right\rangle$.

(v) The magnetization is Fourier transformed to obtain a shifted version of the desired cwESR spectrum centered at the origin instead of the Larmor frequency $\omega_{0}$, as given by Eq. (10.28).

\subsection{MSMs in Service of cw-ESR of Biomolecules}

In this section, the classical molecular dynamics described in Sect. 10.3 and the quantum spin dynamics of Sect. 10.4 are integrated with the purpose of calculating $\mathrm{cw}-\mathrm{ESR}$ spectra from MD simulations. The methodology is applied to T4 Lysozyme in Sect. 10.5.3, for which multifrequency cw-ESR spectra from 72R1 and 131R1 are available. Before presenting this application, however, we start in Sect. 10.5.1 by analyzing the results of a simple analytical model designed to illustrate the influence of motional time scales on the spectral line shape. This analysis helps provide a deeper understanding of the sensitivity and demands of cw-ESR on the time scales and duration of the classical molecular motions, which is discussed in Sect. 10.5.2.

\subsubsection{Coupling Between Markov State Dynamics and Spin Relaxation}

Rather than treating the problem in full generality, the effect of MSM relaxation rates on the spectrum will first be illustrated through the simplest possible example of coupling between a two-state MSM and a two-level spin system. This situation, known as chemical exchange, does arise naturally in magnetic resonance, especially NMR. In our case, it can be reached after a few simplifying assumptions.

For the sake of simplicity, we consider a spin label system in which the electron spin is not coupled to any nuclear spin (unlike a real nitroxide spin label). This leaves us with an ensemble of independent spin- $1 / 2$ systems. In this case, the coherence matrix $\rho_{+}(t)$ becomes a scalar, which is in fact equal to $M_{+}(t)$ (cf. (10.18)). Also, the hyperfine contribution to $H_{z}(t)$ in (10.23) is not present so $H_{z}(t)=\left|\gamma_{\mathrm{e}}\right| B G_{z z}^{\mathrm{L}}(t) / 2$ is also a scalar. With these simplifications the evolution equation (10.22) reduces to

$$
\dot{M}_{+}(t)=\mathrm{i}\left|\gamma_{\mathrm{e}}\right| B G_{z z}^{\mathrm{L}}(t) M_{+}(t)=\mathrm{i} \omega(t) M_{+}(t),
$$

where the last equality defines the precession frequency $\omega(t)$. 
To incorporate molecular motion, we assume that the molecules to which the spins are attached can exist in two different conformations that exchange in a random manner via a hopping process. The conformations are taken to be magnetically distinguishable in the sense that the precession frequency $\omega(t)$ in (10.33) is equal to $\omega_{1}$ in one of the conformations and to $\omega_{2}$ in the other, with $\omega_{1} \neq \omega_{2}$. If $k_{+}$denotes the probability of transition from state 1 to 2 per unit time and $k_{-}$ denotes the probability of transition from state 2 to 1 per unit time, the rate matrix for this twostate MSM is

$$
K=\left[\begin{array}{cc}
-k_{+} & k_{+} \\
k_{-} & -k_{-}
\end{array}\right] .
$$

Using matrix notation, the Master equation (10.10) can be written as

$$
\langle\dot{p}(t)|=\langle p(t)| K,
$$

where $\langle p(t)|=\left[p_{1}(t), p_{2}(t)\right]$, and $p_{1}$ and $p_{2}$ are the probabilities for the chain to be in states 1 and 2, respectively. The left eigenvector of $K$ with eigenvalue zero is the equilibrium (row) vector $\langle\pi|=\left[\pi_{1}, \pi_{2}\right]$. The corresponding right eigenvector is the (column) vector $|1\rangle=[1,1]^{\top}$. The only non-zero eigenvalue of $K$ is $-k$, where $k \equiv k_{+}+k_{-}$is the sole relaxation time scale in this problem. (For comparison, the lifetimes of the two states are $v_{1}=1 / k_{+}$and $v_{2}=1 / k_{-}$, according to (10.11).)

In cw-ESR one detects the transverse magnetization $\mathscr{M}_{+}(t)$ of the whole ensemble and not the probabilities of the two states. Let $M_{1}$ and $M_{2}$ denote the transverse magnetizations of the molecules in the two conformations weighted by the respective probabilities $p_{1}$ and $p_{2}$. In terms of the weighted magnetization (row) vector $\langle M(t)|=\left[M_{1}(t), M_{2}(t)\right]$, the magnetization averaged over the ensemble of molecules is $\mathscr{M}_{+}(t)=M_{1}(t)+M_{2}(t)=\langle M(t) \mid 1\rangle$. As has been shown already by Anderson [2] and Kubo $[28,29]$, the evolution of the probability weighted magnetization vector is given by the joint dynamics of oscillatory motion (10.33) and exchange between the two states (10.35):

$$
\langle\dot{M}(t)|=\langle M(t)|(\mathrm{i} \Omega+K) .
$$

Here, $K$ is the transition rate matrix from (10.34) and

$$
\Omega=\left[\begin{array}{cc}
\omega_{1} & 0 \\
0 & \omega_{2}
\end{array}\right]
$$

is a matrix containing the state-dependent precession frequencies along its main diagonal. For an equilibrated ensemble of spins with unit transverse magnetization the appropriate initial condition is $\langle M(0)|=\langle\pi|$. From (10.13), the cwESR spectrum is the Laplace transform of the transverse magnetization evaluated at $\mathrm{i} \omega$. Taking the Laplace transform of both sides of the KuboAnderson equation (10.36), using the initial condition of $\langle M(t)|$, and taking an inner product with $|1\rangle$, leads to the following expression for the spectrum: $\mathscr{S}(\omega)=\left\langle\pi\left|(\mathrm{i} \omega-\mathrm{i} \Omega-K)^{-1}\right| 1\right\rangle$. Differentiation with respect to $\omega$, in analogy with (10.14), yields the derivative spectrum

$$
\frac{\mathrm{d} \mathscr{S}(\omega)}{\mathrm{d} \omega}=-\mathrm{i}\left\langle\pi\left|(\mathrm{i} \omega-\mathrm{i} \Omega-K)^{-2}\right| 1\right\rangle .
$$

As a numerical example, we choose $\omega_{1}=30$, $\omega_{2}=60$ and $k_{+}=k / 3, k_{-}=2 k / 3$, where the parameter $k$ allows us to vary the exchange rates from slow to fast. This choice of $k_{+}$and $k_{-}$implies that $\pi_{1}=2 / 3$ and $\pi_{2}=1 / 3$ due to detailed balance. The calculated derivative spectra with the relaxation rate $k$ ranging from 10 to 60 are shown in Fig. 10.8. For clarity, only the real parts of the complex spectra are plotted. From the figure, it is seen that for the slow exchange rate $(k=10)$ the spectrum consists of two lines centered at the two precession frequencies. The different intensity of the lines reflects differences in the equilibrium probabilities of the two conformations. When the exchange rate increases $(k=15)$ the centers of the two lines approach each other. At the same time the lines get broader. The approach and broadening of the lines leads to their eventual merger with further increase in the exchange rate $(k=30)$. After that point, the spectrum consists of only one line. Upon further speed up of the exchange $(k=60)$ the center of the single line shifts and its width decreases. For even faster rates of exchange (not shown) there is only one very narrow line centered at the average frequency $\pi_{1} \omega_{1}+\pi_{2} \omega_{2}$, which is equal to $40 \mathrm{in}$ our numerical example. 


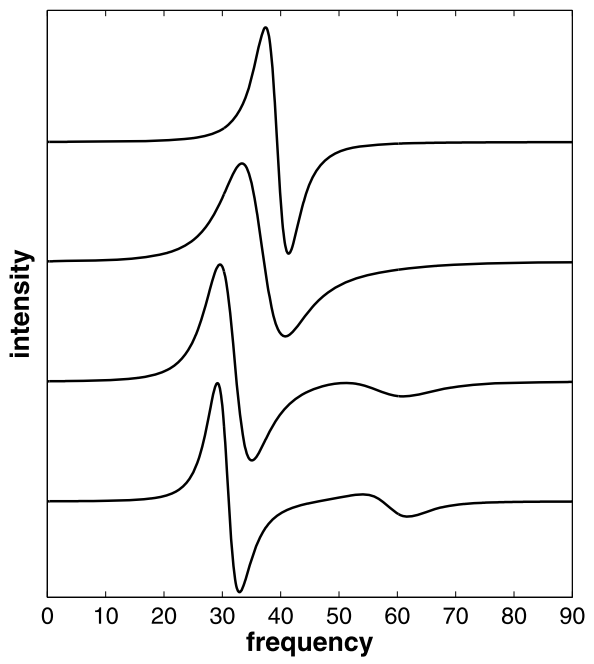

Fig. 10.8 Derivative spectra simulated using Eq. (10.38) with (from bottom to top) $k=10,15,30,60$. For clarity, the spectra are shifted along the vertical axis, which corresponds to the intensity of the spectrum in arbitrary units

In summary, this simple example illustrates that for motions with rates much faster than the spectral width, $W=\left|\omega_{1}-\omega_{2}\right|$, the spectrum reflects the average of the magnetic properties. In contrast, for motions with rates much slower than the spectral width the magnetic properties do not experience averaging due to the dynamics. For intermediate exchange rates the spectral line shape is complex and its detailed structure shows great sensitivity to the rate of exchange. It is exactly in this motional regime that explicit simulations with stochastic models become necessary for the proper interpretation of the experimental situation. All the spectra in Fig. 10.3, for example, fall in this regime.

\subsubsection{Time Scales of the cw-ESR Experiment}

The width of the cw-ESR spectrum depends on the field at which the experiment is performed. For $B=0.35 \mathrm{~T}$ the hyperfine contribution to the propagator $U(t, \Delta t)$ in (10.32) dominates over the Zeeman contribution. Thus, at X-band the width is determined by the $A_{z z}$ component of the hyperfine tensor as $W \approx 2 A_{z z} \approx 90 \mathrm{G}$. When the strength of the magnetic field is increased to $B=3.4 \mathrm{~T}$, the anisotropy of the $g$ tensor (multiplied by the field) is comparable to $2 A_{z z}$. Hence, the Zeeman and hyperfine interactions contribute almost equally to the spectral width, yielding $W \approx 180 \mathrm{G}$. At even higher fields, the spectral width is completely dominated by the anisotropy of the $g$ tensor, which increases linearly with the field strength. Hence, for $B=6.1 \mathrm{~T}$ the spectral width can be estimated as $W \approx 320 \mathrm{G}$.

The relationship between a signal and its Fourier transform implies that the width of a cwESR spectrum, $W$, is inversely proportional to the maximum time step, $\Delta t$, with which the classical dynamics should be followed: $\Delta t=2 \pi / W$. A similar inverse proportionality holds between the total duration of a dynamical trajectory, $T$, and the desired resolution of the cw-ESR spectrum, $\Delta \omega: T=2 \pi / \Delta \omega$. When the frequency axis is reported in units of magnetic field, like in Fig. 10.3, the conversion between magnetic field and angular frequency given in (10.25) needs to be employed. With $\gamma_{\mathrm{e}}$ as given under Eq. (10.1) and $G_{0} \approx 1$, from $\mathbf{g}$ in (10.3) and the definition (10.2), we conclude that 1 Gauss corresponds to a time scale of roughly $360 \mathrm{~ns}$.

Using this conversion factor and the spectral widths estimated above, it can be deduced that for the simulation of cw-ESR spectra at X-band, the magnetization should be known roughly every $\Delta t \lesssim 4 \mathrm{~ns}$. The maximum allowable time step decreases to $\Delta t \lesssim 2 \mathrm{~ns}$ for W-band and $\Delta t \lesssim 1 \mathrm{~ns}$ for G-band spectra. These estimates of $\Delta t$ reflect the temporal resolution with which the FID of the transverse magnetization needs to be known. In the simulation of cw-ESR spectra according to the motional model (10.8), the time step of the numerical integration has to be small enough to faithfully follow not only the FID but the rotational and MSM dynamics as well. Thus, the simulated stochastic process may impose additional demands on the temporal resolution, further reducing the values of $\Delta t$.

An extreme example of the discrepancy between the $\Delta t$ required to follow the decay of the magnetization and the time step of the classical dynamics emerges in the approach (10.6), since the trajectories coming from atomistic MD simulations are typically sampled about every $\delta t=$ 
1 ps. In principle, one could use every snapshot from the trajectories and integrate the spin dynamics with this time step according to (10.31). However, the above estimates of $\Delta t$ indicate that such an approach is unnecessary and wasteful. One option is to decimate the MD trajectories and use snapshots separated by about a thousand steps $[12,53]$. An alternative that we prefer, which follows from the simple example in Sect. 10.5.1 and can be justified rigorously [48, 49], is to average the magnetic tensors over a time window $\Delta t$ $(\Delta t \gg \delta t)$ along each MD trajectory.

For the motional model (10.8), the same logic allows us to pre-average the magnetic tensors over the fast librations of the spin label, visible in the time-traces of its dihedral angles in Fig. 10.5. Using the fragments of the MD trajectories assigned to a given Markovian (macro)state, the magnetic tensors were averaged over the dynamics of $\mathrm{N}$ relative to $\mathrm{M}$ for each state. Such preaveraging not only reduces the effective diagonal values of the magnetic tensors in the nitroxidefixed frame (cf. (10.3)), but also leads to nitroxide frames which have state-dependent orientation relative to the protein frame. In fact, the statedependent "nitroxide frame" calculated in this way is different for the $\mathbf{g}$ and $\mathbf{A}$ tensors since their anisotropies average differently.

The conversion between magnetic field and time implies that a for a spectral resolution of $\Delta \omega \approx 1 \mathrm{G}$ the FID has to be followed for $T \approx$ $360 \mathrm{~ns}$, which gives the necessary duration of a single stochastic trajectory. For spectra broadened by the molecular motion, like the ones shown in Fig. 10.3, a less fine resolution should be sufficient, thus bringing $T$ down by a factor of 3 to 4 ( $T \approx 90-120 \mathrm{~ns}$ ). The time scale $T$ implied by the spectral resolution should be compared with the relaxation time scale $\tau_{1} \gtrsim 100 \mathrm{~ns}$ associated with the rare transition of the disulfide dihedral angle $\chi_{3}$ between the two stable conformations $\mathrm{m}$ and $\mathrm{p}$ (Sect. 10.3.2). Since $\tau_{1}$ falls beyond the time scale relevant for cw-ESR experiments at X-band and especially at higher frequencies, we conclude that its precise value is immaterial for the calculation of such spectra. The example in Sect. 10.5.1 implies that cw-ESR spectra can be simulated as a linear superposition
Table 10.4 Tumbling time scales, $\tau_{D}=1 / 6 D$, associated with diffusion coefficients $D$

\begin{tabular}{lllll}
\hline$D / 10^{6} \mathrm{~s}^{-1}$ & 10 & 18 & 25 & 40 \\
\hline$\tau_{D} / \mathrm{ns}$ & 17 & 9.3 & 6.7 & 4.2 \\
\hline
\end{tabular}

of the separate contributions from the $\mathrm{m}$ and $\mathrm{p}$ conformations of the spin label R1 weighted by their relative populations. This justifies the use of umbrella sampling to determine the populations of the $\mathrm{m}$ and $\mathrm{p}$ states (Sect. 10.3.2), without any knowledge about the rate of their interconversion.

The considerations in the previous paragraphs suggest that cw-ESR experiments are very sensitive to motions in the time window of about 2-50 ns, which constitutes the middle of the estimated spectral time scale. Additional dynamics falling outside this time window are expected to have a lesser effect on the spectral line shape and be largely inaccessible on the background of the 2-50 ns motions. The time scales corresponding to the DNA tumbling rates used in the spectral simulations of Fig. 10.3 are compiled in Table 10.4. Clearly, they all fall in the window where the cw-ESR spectra are expected to be strongly affected. The additional diffusion coefficient in Table 10.4, with $\tau_{D}=9.3 \mathrm{~ns}$, corresponds to the tumbling of the protein T4 Lysozyme in solution. On the basis of this time scale, we expect that the rotational diffusion of the protein has to be explicitly taken into account for quantitative simulation of cw-ESR spectra from spin-labeled T4 Lysozyme, to be examined in Sect. 10.5.3.

Let us use the developed intuition to rationalize the qualitative differences between the spectra shown in Fig. 10.3. As already mentioned, at X-band the effect of the $g$ tensor is negligible and the spectral line shape is heavily dominated by the hyperfine tensor. Since $\mathbf{A}$ is an axial tensor, any differences in the rate of mixing of its components $A_{x x}^{\mathrm{N}}=A_{y y}^{\mathrm{N}}$ by the rotational diffusion of the macromolecule is inconsequential. This is the reason the spectra simulated using $D_{\|}=40 \times 10^{6} \mathrm{rad}^{2} / \mathrm{s}$ (black) and $D_{\|}=10 \times 10^{6} \mathrm{rad}^{2} / \mathrm{s}$ (blue) appear identical at $B=0.34 \mathrm{~T}$ (Fig. 10.3A). In contrast, spectra at $B=0.34 \mathrm{~T}$ are strongly influenced by differences in the rate of mixing between the $A_{x x}^{\mathrm{N}}=A_{y y}^{\mathrm{N}}$ 
components of the hyperfine tensor and $A_{z z}^{\mathrm{N}}$, as illustrated by the spectra in Fig. 10.3A simulated using $D_{\perp}=10 \times 10^{6} \mathrm{rad}^{2} / \mathrm{s}$ (black) and $D_{\perp}=$ $25 \times 10^{6} \mathrm{rad}^{2} / \mathrm{s}$ (red). At W-band, the $g$-tensor anisotropy influences the spectral line shape as much as the hyperfine tensor. Because the $g$ tensor of the nitroxide distinguishes between all the three directions of the coordinate axes, the highfield spectra are sensitive to the rates of mixing induced by both $D_{\|}$and $D_{\perp}$, as clearly seen in Fig. 10.3B.

By dwelling further on the spectra of Fig. 10.3 we hope to have convinced the reader that cwESR spectra are very sensitive to both the directionality and the magnitude of the molecular motions (reflecting the local structure) when they fall in the spectral time scale of the experiment. When the dynamics is either faster or slower than the spectral time scale, the spectrum still carries information about the average magnetic properties or the populations of the slowly exchanging conformations, as demonstrated by the example in Sect. 10.5.1. Therefore, quantitative comparison with cw-ESR spectra at several different frequencies, from X- to W- to G-band, should provide an unprecedented check on the structural and dynamical aspects of the internal spin-label dynamics captured by the MD simulations of $\mathrm{T} 4$ Lysozyme.

\subsubsection{Multifrequency cw-ESR Spectra of Spin-Labeled T4 Lysozyme}

Although the MSMs of the spin label R1 at positions 72 and 131 on T4L were constructed on the basis of the time-series of the five spin label torsion angles (Sect. 10.3.2), the influence of the environment is implicitly incorporated in two ways. First, the electrostatic and van der Waals interactions with the protein and the solvent molecules dictate which rotameric states of the spin label are populated and to what extent. Due to the internal flexibility and amphiphilic nature of the spin label, the populations of its conformations are sensitive to the subtle balance between various interactions, and are hard to predict on the basis of simplified steric and hydrodynamic arguments
$[47,54]$. Second, explicit protein and solvent dynamics on time scales up to about 100 ps was used to calculate pre-averaged magnetic tensors for each of the states of the MSMs, as described in Sect. 10.5.2. Therefore, in addition to the exchange between the rotamers, the rattling of the nitroxide in the solvent cage and the local thermal fluctuations of the protein backbone (as opposed to larger scale conformational changes, e.g. partial unfolding of the helices, etc.), are implicitly accounted for in the spectral simulations presented next.

$\mathrm{Cw}-\mathrm{ESR}$ spectra for $72 \mathrm{R} 1$ and $131 \mathrm{R} 1$ in T4 Lysozyme were calculated for three different magnetic field strengths $-0.33 \mathrm{~T}, 3.4 \mathrm{~T}$ and $6.1 \mathrm{~T}$-according to the motional model (10.8). Isotropic rotational diffusion with diffusion constant $D=18 \times 10^{6} \mathrm{rad} / \mathrm{s}$ (Table 10.4) was used to account for the global tumbling of the protein. In Figs. 10.9 and 10.10, the spectra calculated by using separately the $\mathrm{m}$ and $\mathrm{p}$ subblocks of the estimated transition probability matrices are shown on the left, and the final spectra obtained by linearly mixing the FID decays of the $m$ and $p$ states are shown on the right. For the three fields, the spectra from the $m$ and $p$ conformations of 131R1 are quite similar to each other and to the experimental spectra, with the difference increasing slightly with the increase of the field (Fig. 10.10). The agreement between the calculated and the experimental spectra is remarkably good over the entire field range. In the case of $72 \mathrm{R} 1$, the $\mathrm{m}$ and $\mathrm{p}$ contributions to the spectra are markedly different, with the latter being consistently more similar to the experimental spectrum for all the three field strengths (Fig. 10.9). At $0.33 \mathrm{~T}(9 \mathrm{GHz})$, the p component by itself is basically identical to the experimental spectrum, whereas adding $27 \%$ of the $\mathrm{m}$ component is essential for the good agreement at the two higher fields.

By changing the ESR frequency from $9 \mathrm{GHz}$ to $170 \mathrm{GHz}$ the time window of sensitivity of the experiment is changed by about an order of magnitude. Also, whereas at $9 \mathrm{GHz}$ the spectrum is dominated by the hyperfine tensor, at $95 \mathrm{GHz}$ the contribution of the $g$ tensor becomes more significant, and eventually dominates at $170 \mathrm{GHz}$. Therefore, the quantitative 
Fig. 10.9 Experimental spectra of $72 \mathrm{R} 1$ at $22{ }^{\circ} \mathrm{C}$ (black). Left: Calculated spectra of conformations $\mathrm{m}$ (blue) and p (red); Right: Spectra calculated by mixing the $m$ and $p$ conformations in the specified ratio (green). Simulation parameters given in Ref. [50]
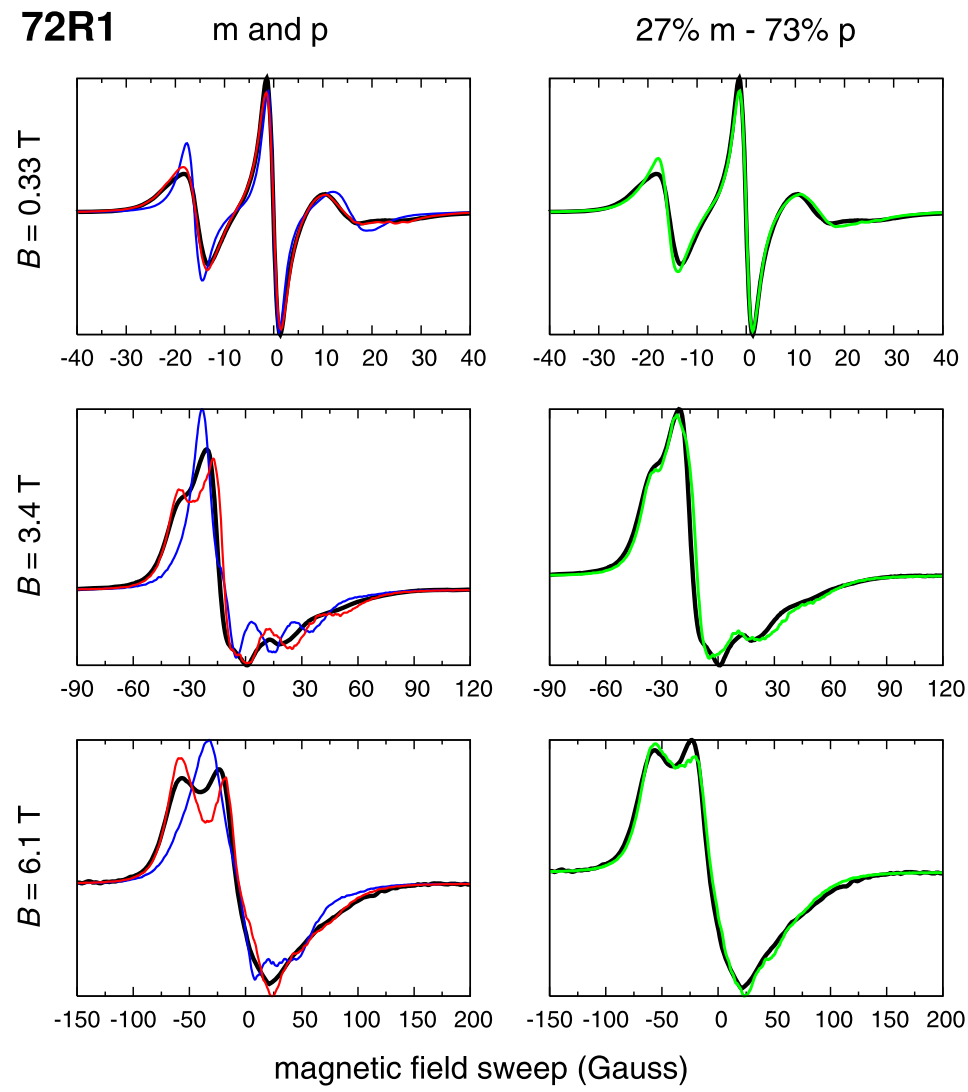

agreement of the calculated spectra with experiment over the 9-170 GHz range is strongly suggestive that the dynamics of the spin label in the computer simulations is quite similar to the real underlying dynamics.

A unique dynamical model cannot be inferred on the basis of $9 \mathrm{GHz}$ spectra alone. Multifrequency ESR analysis attempts to address this issue by placing additional restrictions on the nature of the microscopic dynamics. The constraints presented by multifrequency spectra are important, e.g., a single diffusional MOMD model is unable to simultaneously fit both the low and high frequency spectra from T4L $[3,33]$. Achieving a simultaneous agreement is challenging even for state-of-the-art fitting approaches based on the SRLS model, which also raise the question of uniqueness of fit, even with the additional restrictions imposed by multifrequency cw-ESR [56]. It is notable that the present results, which have yielded spectra in excellent agreement with ex- periment at three different frequencies, were generated from a single microscopic model without any fitting parameters or ad hoc empirical adjustement of the model.

\subsection{Summary and Future Outlook}

A novel methodological framework was elaborated for the purpose of simulating $\mathrm{cw}$-ESR spectra of spin labeled proteins from all-atom MD trajectories [49]. Within this framework, the information from multiple independent MD trajectories is employed to construct an MSM of the R1 dynamics in the space of its five dihedral angles. Using the transition probability matrix of the MSM determined from the MD simulations, long stochastic trajectories including rotational diffusion are generated to simulate realistic cw-ESR spectra [48]. This framework was used to study the conformations and dynamics of 
Fig. 10.10 Experimental spectra of $131 \mathrm{R} 1$ at $22{ }^{\circ} \mathrm{C}$ (black). Left: Calculated spectra of conformations $\mathrm{m}$ (blue) and p (red); Right: Spectra calculated by mixing the $m$ and $p$ conformations in the specified ratio (green). Simulation parameters given in Ref. [50]
131R1 $m$ and $p$
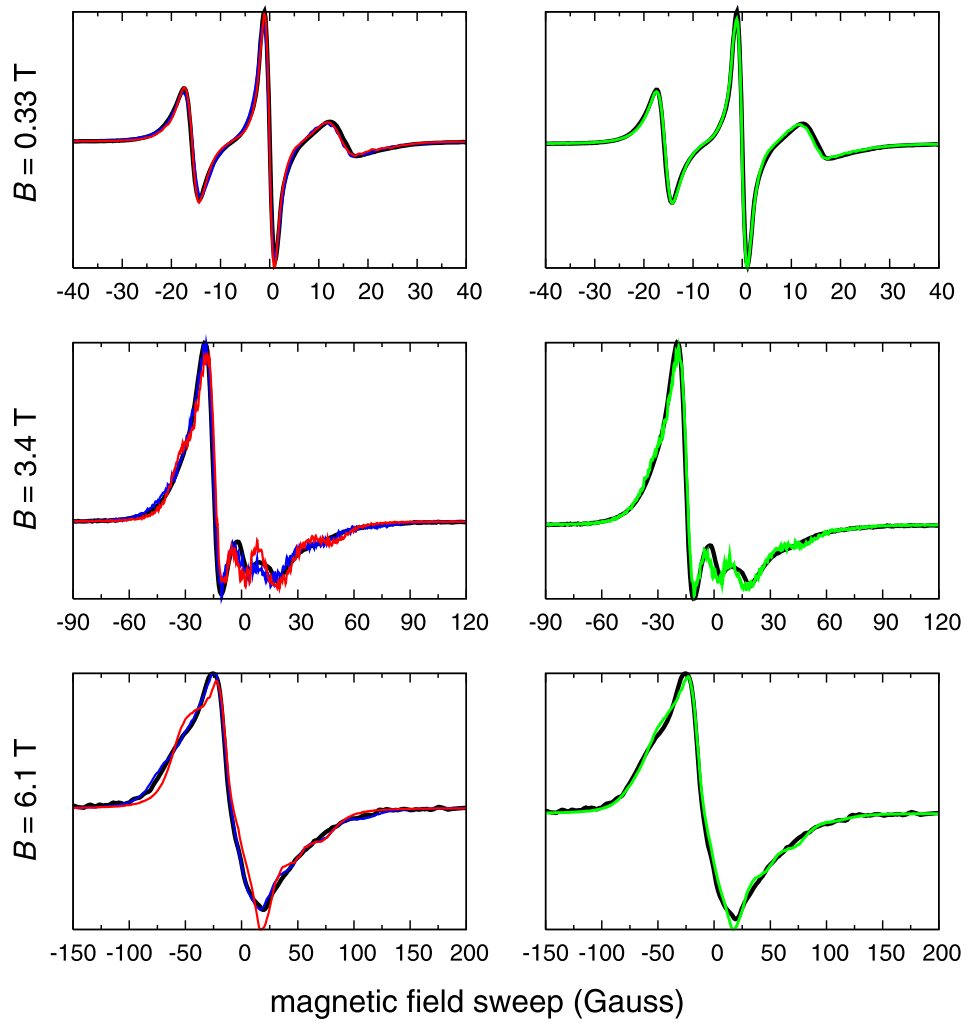

$55 \% m-45 \% p$
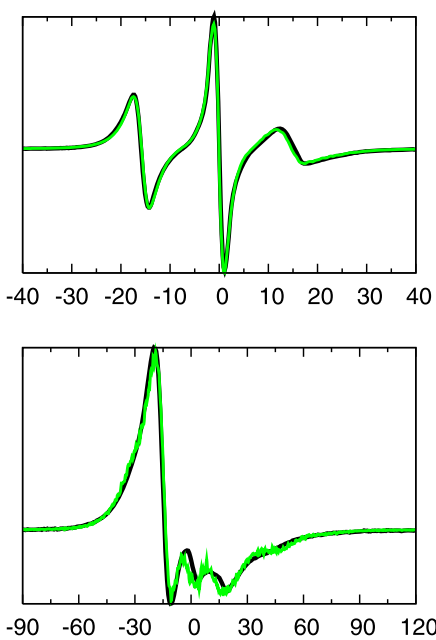

the spin label R1 at positions 72 and 131 in $\mathrm{T} 4$ Lysozyme. For the first time, very good agreement with multifrequency cw-ESR experiments at three different magnetic field strengths was obtained. The atomically-detailed picture of the spin label emerging from the MD simulations helps to unify spectroscopic and crystallographic data and provides useful insight into their molecular origins.

The MSMs constructed from the MD simulations can be viewed as a natural extension of the multicomponent SRLS model, in which the spin label dynamics is modeled as a linear superposition of several independent motional modes, each characterized by a microscopic ordering potential and a rotational diffusion tensor. It is our hope that the overall perspective developed from the MD simulations can help design better motional models tailored to the specific spin label and biomolecule to which it is attached. Going beyond a universal, generic stochastic model is expected to be of crucial importance, given the extensive applications of SDSL to diverse biological systems and the increased availability of high-field ESR.

Accurate calculations of multifrequency cwESR spectra by mapping MD trajectories onto MSMs are extremely challenging because they require that a whole host of molecular motions be accounted for correctly, not only in terms of their amplitudes and resulting equilibrium populations, but also in terms of their dynamical timescales. While the observables from many experimental methods (e.g. FRET, NMR, hydrogen exchange) are often dominated by one or a few relaxation modes, multifrequency $\mathrm{cw}$-ESR spectra provides perhaps one of the rare applications of the methodology where a large fraction of the set of eigenvalues and eigenvectors of the MSM rate matrix is truly put to the test. In this context, the present success in quantitatively reproducing experimental multifrequency $\mathrm{cw}-\mathrm{ESR}$ spec- 
tra, achieved without any empirical adjustment, is truly remarkable. From a broader perspective, the ESR/MSM methodology elaborated here offers a powerful route to test and validate the ability of existing force fields to reproduce both structural and dynamical aspects of the molecular motions as reported by the spin label. It will be of great interest to carry out additional ESR/MSM simulations to cover a range of experimental conditions (e.g., temperature, viscosity) to further test their ability to predict $\mathrm{cw}$-ESR spectra under different conditions.

\section{References}

1. Abragam A (1961) The principles of nuclear magnetism. Oxford University Press, New York

2. Anderson PW (1954) A mathematical model for the narrowing of spectral lines by exchange or motion. J Phys Soc Jpn 9(3):316-339

3. Barnes JP, Liang Z, Mchaourab HS, Freed JH, Hubbell WL (1999) A multifrequency electron spin resonance study of T4 Lysozyme dynamics. Biophys J 76(23):298-3306

4. Beier C, Steinhoff HJ (2006) A structure-based simulation approach for electron paramagnetic resonance spectra using molecular and stochastic dynamics simulations. Biophys J 91:2647-2664

5. Bennati M, Prisner TF (2005) New developments in high field electron paramagnetic resonance with applications in structural biology. Rep Prog Phys 68(2)

6. Borbat PP, Costa-Filho AJ, Earle KA, Moscicki JK, Freed JH (2001) Electron spin resonance in studies of membranes and proteins. Science 291:266-269

7. Budil DE, Lee S, Saxena S, Freed JH (1996) Nonlinear-least-squares analysis of slow-motion EPR spectra in one and two dimensions using a modified Levenberg-Marquardt algorithm. J Magn Reson, Ser A 120:155-189

8. Budil DE, Sale KL, Khairy KA, Fajer PG (2006) Calculating slow-motional electron paramagnetic resonance spectra from molecular dynamics using a diffusion operator approach. J Phys Chem A 110:37033713

9. Cekan P, Sigurdsson ST (2009) Identification of single-base mismatches in duplex DNA by EPR spectroscopy. J Am Chem Soc 131(50):18,054-18,056

10. Columbus L, Hubbell WL (2002) A new spin on protein dynamics. Trends Biochem Sci 27:288-295

11. Columbus L, Kalai T, Jeko J, Hideg K, Hubbell WL (2001) Molecular motion of spin labeled side chains in $\alpha$-helices: analysis by variation of side chain structure. Biochemistry 40:3828-3846
12. DeSensi SC, Rangel D, Lybrand TP, Hustedt EJ (2008) The calculation of nitroxide cw-EPR spectra from Brownian dynamic trajectories and molecular dynamics simulations. Biophys J 94(10):3798-3809

13. Earle KA, Dzikovski B, Hofbauer W, Moscicki JK, Freed JH (2005) High-frequency ESR an ACERT. Magn Reson Chem 43:S256-S266

14. Eviatar H, van Faassen E, Levine Y, Hoult D (1994) Time-domain simulation of ESR spectra of nitroxide spin probes. Chem Phys 181:369-376

15. Eviatar H, van der Heide U, Levine YK (1995) Computer simulations of the electron spin resonance spectra of steroid and fatty acid nitroxide probes in bilayer systems. J Chem Phys 102:3135-3145

16. Fanucci GE, Cafiso DS (2006) Recent advances and applications of site-directed spin labeling. Curr Opin Struct Biol 16:644-653

17. Fedchenia II, Westlund PO, Cegrell U (1993) Brownian dynamics simulation of restricted molecular diffusion. The symmetric and deformed cone models. Mol Simul 11:373-393

18. Fleissner MR, Cascio D, Hubbell WL (2009) Structural origin of weakly ordered nitroxide motion in spin-labeled proteins. Protein Sci 18(5):893-908

19. Freed JH (1976) Theory of slow motional ESR spectra for nitroxides. In: Berliner LJ (ed) Spin labeling: theory and application. Academic Press, New York, pp 53-132

20. Freed JH (2000) New technologies in electron spin resonance. Annu Rev Phys Chem 51:655-689

21. Guo Z, Cascio D, Hideg K, Kalai T, Hubbell WL (2007) Structural determination of nitroxide motion in spin-labeled proteins: tertiary contact and solventinaccessible sites in helix G of T4 Lysozyme. Protein Sci 16:1069-1086

22. Hakansson P, Westlund PO, Lindahl E, Edholm O (2001) A direct simulation of EPR slow-motion spectra of spin labelled phospholipids in liquid crystalline bilayers based on a molecular dynamics simulation of the lipid dynamics. Phys Chem Chem Phys 3:53115319

23. Halle B (2009) The physical basis of model-free analysis of NMR relaxation data from proteins and complex fluids. J Chem Phys 131(22):224,507-224,522

24. Hartigan JA (1975) Clustering algorithms. Wiley, New York

25. Jiao D, Barfield M, Combariza JE, Hruby VJ (1992) $\mathrm{Ab}$ initio molecular orbital studies of the rotational barriers and the sulfur-33 and carbon-13 chemical shieldings for dimethyl disulfide. J Am Chem Soc 114(10):3639-3643

26. Klare JP, Steinhoff HJ (2009) Spin labeling EPR. Photosynth Res 102(2-3):377-390

27. Krstic I, Endeward B, Margraf D, Marko A, Prisner TF (2012) Structure and dynamics of nucleic acids. Top Curr Chem 321:159-198

28. Kubo R (1954) Note on the stochastic theory of resonance absorption. J Phys Soc Jpn 9(6):935-944

29. Kubo R (1969) A stochastic theory of line shape. Adv Chem Phys 15:101-127 
30. Kuprusevicius E, White G, Oganesyan VS (2011) Prediction of nitroxide spin label EPR spectra from MD trajectories: application to myoglobin. Faraday Discuss 148:283-298

31. Langen R, Oh KJ, Cascio D, Hubbell WL (2000) Crystal structures of spin labeled T4 Lysozyme mutants: implications for the interpretation of EPR spectra in terms of structure. Biochemistry 39:8396-8405

32. Levitt MH (2008) Spin dynamics: basics of nuclear magnetic resonance, 2nd edn. Wiley, Chichester

33. Liang Z, Lou Y, Freed JH, Columbus L, Hubbell WL (2004) A multifrequency electron spin resonance study of T4 Lysozyme dynamics using the slowly relaxing local structure model. J Phys Chem B 108:17,649-17,659

34. Maragakis P, Lindorff-Larsen K, Eastwood MP, Dror RO, Klepeis JL, Arkin IT, Jensen MO, Xu H, Trbovic N, Friesner RA, Palmer AG, Shaw DE (2008) Microsecond molecular dynamics simulation shows effect of slow loop dynamics on backbone amide order parameters of proteins. J Phys Chem B 112(19):6155-6158

35. Mchaourab HS, Kalai T, Hideg K, Hubbell WL (1999) Motion of spin-labeled side chains in T4 Lysozyme: effect of side chain structure. Biochemistry 38:2947-2955

36. Mchaourab HS, Lietzow MA, Hideg K, Hubbell WL (1996) Motion of spin-labeled side chains in T4 Lysozyme. Correlation with protein structure and dynamics. Biochemistry 35:7692-7704

37. Mchaourab HS, Steed PR, Kazmier K (2011) Toward the fourth dimension of membrane protein structure: insight into dynamics from spin-labeling EPR spectroscopy. Structure 19(11):1549-1561

38. Meirovitch E, Nayeem A, Freed JH (1984) Analysis of protein-lipid interactions based on model simulations of electron spin resonance spectra. J Phys Chem $88: 3454-3465$

39. Norris JR (1997) Markov chains. Cambridge University Press, Cambridge

40. Polimeno A, Freed JH (1993) A many-body stochastic approach to rotational motions in liquids. Adv Chem Phys 83:89-210

41. Polimeno A, Freed JH (1995) Slow motional ESR in complex fluids: the slowly relaxing local structure model of solvent cage effects. J Phys Chem 99:10,995-11,006

42. Rabiner LR (1989) A tutorial on hidden Markov models and selected applications in speech recognition. Proc IEEE 77:257-286

43. Redfield AG (1957) On the theory of relaxation processes. IBM J Res Dev 1:19-31
44. Robinson BH, Slutsky LJ, Auteri FP (1992) Direct simulation of continuous wave electron paramagnetic resonance spectra from Brownian dynamics trajectories. J Chem Phys 96:2609-2616

45. Roux B (1994) The calculation of the potential of mean force using computer simulations. Comput Phys Commun 91:275-282

46. Schneider DJ, Freed JH (1989) Spin relaxation and motional dynamics. Adv Chem Phys 73:387-527

47. Sezer D, Freed JH, Roux B (2008) Parametrization, molecular dynamics simulation, and calculation of electron spin resonance spectra of a nitroxide spin label on a polyalanine alpha-helix. J Phys Chem B 112(18):5755-5767

48. Sezer D, Freed JH, Roux B (2008) Simulating electron spin resonance spectra of nitroxide spin labels from molecular dynamics and stochastic trajectories. J Chem Phys 128(16):165,106-165,116

49. Sezer D, Freed JH, Roux B (2008) Using Markov models to simulate electron spin resonance spectra from molecular dynamics trajectories. J Phys Chem B 112(35):11,014-11,027

50. Sezer D, Freed JH, Roux B (2009) Multifrequency electron spin resonance spectra of a spin-labeled protein calculated from molecular dynamics simulations. J Am Chem Soc 131(7):2597-2605

51. Sezer D, Sigurdsson ST (2011) Simulating electron spin resonance spectra of macromolecules labeled with two dipolar-coupled nitroxide spin labels from trajectories. Phys Chem Chem Phys 13(28):12,78512,797

52. Steinhoff HJ, Hubbell W (1996) Calculation of electron paramagnetic resonance spectra from Brownian dynamics trajectories: application to nitroxide side chains in proteins. Biophys J 71:2201-2212

53. Stoica I (2004) Using molecular dynamics to simulate electronic spin resonance spectra of T4 Lysozyme. J Phys Chem B 108(5):1771-1782

54. Tombolato F, Ferrarini A, Freed JH (2006) Dynamics of nitroxide side chain in spin-labeled proteins. J Phys Chem B 110:26,248-26,259

55. Usova N, Westlund PO, Fedchenia I (1995) Direct simulation of slow-motion electron spin resonance spectra by solving the stochastic Liouville equation in time domain with stochastic dynamics in the form of trajectories. J Chem Phys 103:96-103

56. Zhang Z, Fleissner MR, Tipikin DS, Liang Z, Moscicki JK, Earle KA, Hubbell WL, Freed JH (2010) Multifrequency electron spin resonance study of the dynamics of spin labeled T4 Lysozyme. J Phys Chem B 114(16):5503-5521 